\title{
New Fixed-Target Experiments to Search for Dark Gauge Forces
}

\author{
James D. Bjorken, ${ }^{1}$ Rouven Essig, ${ }^{1}$ Philip Schuster, ${ }^{1}$ and Natalia Toro ${ }^{2}$ \\ ${ }^{1}$ Theory Group, SLAC National Accelerator Laboratory, Menlo Park, CA 94025 \\ ${ }^{2}$ Theory Group, Stanford University, Stanford, CA 94305
}

(Dated: June 3, 2009)

\begin{abstract}
Fixed-target experiments are ideally suited for discovering new $\mathrm{MeV}-\mathrm{GeV}$ mass $U(1)$ gauge bosons through their kinetic mixing with the photon. In this paper, we identify the production and decay properties of new light gauge bosons that dictate fixed-target search strategies. We summarize existing limits and suggest five new experimental approaches that we anticipate can cover most of the natural parameter space, using currently operating $\mathrm{GeV}$-energy beams and well-established detection methods. Such experiments are particularly timely in light of recent terrestrial and astrophysical anomalies (PAMELA, FERMI, DAMA/LIBRA, etc.) consistent with dark matter charged under a new gauge force.
\end{abstract}

\section{NEW GAUGE FORCES}

The interactions of ordinary matter establish that three gauge forces survive to low energies. Two striking features of these forces - electroweak symmetrybreaking at a scale far below the Planck scale and apparent unification assuming low-energy supersymmetry - have driven model-building for a quarter-century. But the strong and electroweak forces need not be the only ones propagating at long distances. Additional forces, under which ordinary matter is neutral, would have gone largely unnoticed because gauge symmetry prohibits renormalizable interactions between Standard Model fermions and the other "dark" gauge bosons or matter charged under them.

There is an important exception to the above claim: new "dark" Abelian forces can couple to Standard Model hypercharge through the kinetic mixing operator $\frac{\epsilon}{2} F_{\mu \nu}^{Y} F^{\prime \mu \nu}$, where $F_{\mu \nu}^{\prime}=\partial_{[\mu} A_{\nu]}^{\prime}$ and $A^{\prime}$ is the dark gauge field [1. If the $A^{\prime}$ is massive, Standard Model matter acquires milli-charges proportional to $\epsilon$ under the massive $A^{\prime}$. Kinetic mixing with $\epsilon \sim 10^{-8}-10^{-2}$ can be generated at any scale by loops of heavy fields charged under both $U(1)^{\prime}$ and $U(1)_{Y}$, and the $A^{\prime}$ can acquire mass through a technicolor or Higgs mechanism. A mass scale near but beneath the weak scale is particularly well-motived $U(1)^{\prime}$ symmetry-breaking may be protected by the same physics that stabilizes the electroweak hierarchy [2. Indeed, if the largest symmetry-breaking effects arise from weak-scale supersymmetry breaking, then the $U(1)^{\prime}$ symmetry breaking scale is naturally suppressed by a loop factor or by $\sqrt{\epsilon}$, leading to $\mathrm{MeV}$ to $\mathrm{GeV}$-scale $A^{\prime}$ masses [2, 3, 4, 5, 6.

An $A^{\prime}$ can be produced in collisions of charged particles with nuclei and can decay to electrons or muons. The production cross-section $\left(\sigma_{A^{\prime}}\right)$ and decay length $(\gamma c \tau)$,

$$
\begin{aligned}
\sigma_{A^{\prime}} & \sim 100 \mathrm{pb}\left(\epsilon / 10^{-4}\right)^{2}\left(100 \mathrm{MeV} / m_{A^{\prime}}\right)^{2} \\
\gamma c \tau & \sim 1 \mathrm{~mm}(\gamma / 10)\left(10^{-4} / \epsilon\right)^{2}\left(100 \mathrm{MeV} / m_{A^{\prime}}\right)
\end{aligned}
$$

vary by ten orders of magnitude for the $\epsilon$ 's and masses $m_{A^{\prime}}$ we consider. This wide range calls for multiple experimental approaches, with different strategies for confronting backgrounds. Beam-dump searches from the 1980's exclude the low-mass and small- $\epsilon$ parameter range, and other data constrains large $\epsilon$. In this paper we suggest five scenarios for fixed-target experiments sensitive to distinct but overlapping regions of parameter space (see Figure 1). Together they can probe six decades in $A^{\prime}$ coupling and three decades in $A^{\prime}$ mass with existing beam energies and intensities.

Dark matter interpretations of recent astrophysical and terrestrial anomalies provide a further impetus to search for new $U(1)$ 's. Annihilation of dark matter charged under a new $U(1)^{\prime}$ into the $A^{\prime}$ can explain the electron and/or positron excesses observed by PAMELA [7, ATIC [8, FERMI 9], and HESS [10, 11] (see e.g. 12, 13, 14, 15, 16, 17, 18, 19]). If the dark matter is also charged under a non-abelian group, then its spectrum naturally implements an inelastic dark matter scenario [20, thereby explaining the annual modulation signal reported by DAMA/LIBRA [21, 22] and reconciling it with the null results of other experiments [12, 20, 23, 24].

In view of these suggestive data and the abundant theoretical speculation surrounding them, insight from new experiments is clearly called for. New probes of weakly mixed $\mathrm{MeV}-\mathrm{GeV} U(1)$ 's directly probe the low-energy structure of these scenarios, where the nature of their interactions is most manifest. As such, the experiments we advocate here are complementary to upcoming gammaray observations (see e.g. [25, 26]) and to the next generation of direct detection experiments [27, 28, 29, 30, 31. that will shed light on the scattering of dark matter.

\section{Direct Tests of Low-Mass Gauge Sectors}

Constraints on new $A^{\prime}$ s and the reach of different experiments are summarized in Figure 1. To begin, low- 

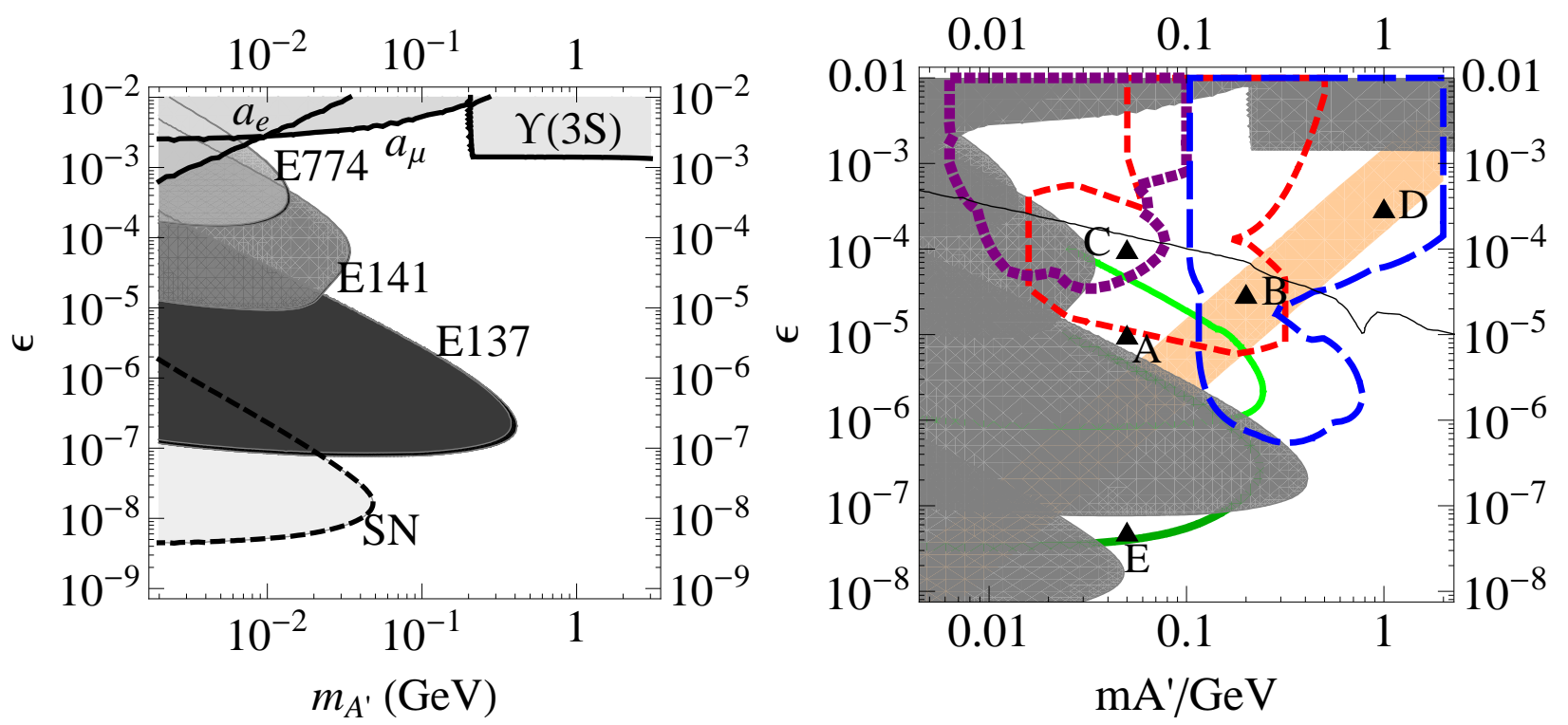

FIG. 1: Left: Existing constraints on an $A^{\prime}$. Shown are constraints from electron and muon anomalous magnetic moment measurements, $a_{e}$ and $a_{\mu}$, the BaBar search for $\Upsilon(3 S) \rightarrow \gamma \mu^{+} \mu^{-}$, three beam dump experiments, E137, E141, and E774, and supernova cooling (SN). These constraints are discussed further in Section III. Right: Existing constraints are shown in gray, while the various lines - light green (upper) solid, red short-dashed, purple dotted, blue long-dashed, and dark green (lower) solid - show estimates of the regions that can be explored with the experimental scenarios discussed in Section IV AIVE respectively. The discussion in IV focuses on the five points labeled "A" through "E". The orange stripe denotes the "D-term" region introduced in section [II A] in which simple models of dark matter interacting with the $A^{\prime}$ can explain the annual modulation signal reported by DAMA/LIBRA. Along the thin black line, the $A^{\prime}$ proper lifetime $c \tau=80 \mu \mathrm{m}$, which is approximately the $\tau$ proper lifetime.

energy $e^{+} e^{-}$colliders are a powerful laboratory for the study of an $A^{\prime}$ with $\epsilon \gtrsim 10^{-4}$ and mass above $\sim 200$ $\mathrm{MeV}$, particularly in sectors with multiple light states [32, 33, 34, 35, 36. Their reach in $\epsilon$ is limited by luminosity and irreducible backgrounds. However, an $A^{\prime}$ can also be produced through bremsstrahlung off an electron beam incident on a fixed target [34. This approach has several virtues over colliding-beam searches: much larger luminosities, of $\mathcal{O}\left(1 \mathrm{ab}^{-1} /\right.$ day $)$ can be achieved, scattering cross-sections are enhanced by nuclear charge coherence, and the resulting boosted final states can be observed with compact special-purpose detectors.

Past electron "beam-dump" experiments, in which a detector looks for decay products of rare penetrating particles behind a stopped electron beam, constrain $\gtrsim 10$ $\mathrm{cm}$ vertex displacements and $\epsilon \gtrsim 10^{-7}$. The thick shield needed to stop beam products limits these experiments to long decay lengths, so thinner targets are needed to probe shorter displacements (larger $\epsilon$ and $m_{A^{\prime}}$ ). However, beam products easily escape thin targets and constitute a challenging background in downstream detectors.

The five benchmark points labeled "A" through "E" in Figure 1 (right) require different approaches to these challenges, discussed in Section IV. We have estimated the reach of each scenario, summarized in Figure 1 (right), in the context of electron beams with $1-6 \mathrm{GeV}$ energies, $\mathrm{nA}-\mu \mathrm{A}$ average beam currents, and run times $\sim 10^{6} \mathrm{~s}$. Such beams can be found for example at the
Thomas Jefferson National Accelerator Facility (JLab), the SLAC National Accelerator Laboratory, the electron accelerator ELSA, and the Mainzer Mikrotron (MAMI).

The scenarios for points $\mathrm{A}$ and $\mathrm{E}$ use $100 \mathrm{MeV}-1 \mathrm{GeV}$ electron beam dumps, with more complete event reconstruction or higher-current beams than previous dump experiments. Low-mass, high- $\epsilon$ regions (e.g. B and C) produce boosted $A^{\prime}$ and forward decay products with $\mathrm{mm}-\mathrm{cm}$ displaced vertices. Our approaches exploit very forward silicon-strip tracking to identify these vertices, while maintaining reasonable occupancy - a limiting factor. At still higher $\epsilon$, no displaced vertices are resolvable and one must take full advantage of the kinematic properties of the signal and background processes, including the recoiling electron, using either the forward geometries of $\mathrm{B}$ and $\mathrm{C}$ or a wider-angle spectrometer (e.g. for point D). Spectrometers operating at various laboratories appear capable of probing this final region.

We focus on the case where the $A^{\prime}$ decays directly to Standard Model fermions, but the past experiments and proposed scenarios are also sensitive (with different exclusions) if the $A^{\prime}$ decays to lighter $U(1)^{\prime}$-charged scalars, and to direct production of axion-like states.

\section{Outline}

In Section II] we summarize the properties of $A^{\prime}$ production through bremsstrahlung in fixed-target colli- 
sions. Constraints from past experiments and from neutrino emission by SN 1987A are presented in Section III. In Section IV] we describe the five new experimental scenarios and estimate the limiting backgrounds. We conclude in Section $\mathrm{V}$ with a summary of the prospects for new experiments. More detailed formulas, which we use to calculate our expected search reaches, and a more detailed discussion of some of the backgrounds, are given in Appendices $\mathrm{A}, \mathrm{B}$, and $\mathrm{C}$.

\section{THE PHYSICS OF NEW $U(1)$ VECTORS IN FIXED TARGET COLLISIONS}

\section{A. Theoretical Preliminaries}

Consider the Lagrangian

$$
\mathcal{L}=\mathcal{L}_{\mathrm{SM}}+\epsilon_{Y} F^{Y, \mu \nu} F_{\mu \nu}^{\prime}+\frac{1}{4} F^{\prime, \mu \nu} F_{\mu \nu}^{\prime}+m_{A^{\prime}}^{2} A^{\prime \mu} A_{\mu}^{\prime},
$$

where $\mathcal{L}_{\mathrm{SM}}$ is the Standard Model Lagrangian, $F_{\mu \nu}^{\prime}=$ $\partial_{[\mu} A_{\nu]}^{\prime}$, and $A^{\prime}$ is the gauge field of a massive dark $U(1)^{\prime}$ gauge group [1. The second term in (3) is the kinetic mixing operator, and $\epsilon \sim 10^{-8}-10^{-2}$ is naturally generated by loops at any mass scale of heavy fields charged under both $U(1)^{\prime}$ and $U(1)_{Y}$; the lower end of this range is obtained if one or both $U(1)$ 's are contained in grandunified (GUT) groups, since then $\epsilon$ is only generated by two-or three-loop GUT-breaking effects.

A simple way of analyzing the low-energy effects of the $A^{\prime}$ is to treat kinetic mixing as an insertion of $p^{2} g_{\mu \nu}-p_{\mu} p_{\nu}$ in Feynman diagrams, making it clear that the $A^{\prime}$ couples to the electromagnetic current of the Standard Model through the photon. This picture also clarifies, for example, that new interactions induced by kinetic mixing must involve a massive $A^{\prime}$ propagator, and that effects of mixing with the $Z$-boson are further suppressed by $1 / m_{Z}^{2}$. Equivalently, one can redefine the photon field $A^{\mu} \rightarrow A^{\mu}+\epsilon A^{\prime \mu}$ as in [37, which removes the kinetic mixing term and generates a coupling $e A_{\mu} J_{\mathrm{EM}}^{\mu} \supset \epsilon e A_{\mu}^{\prime} J_{\mathrm{EM}}^{\mu}$ of the new gauge boson to electrically charged particles (here $\epsilon \equiv \epsilon_{Y} \cos \theta_{W}$ ). Note that this does not induce electromagnetic millicharges for particles charged under the $A^{\prime}$. The parameters of concern in this paper are $\epsilon$ and $m_{A^{\prime}}$.

We now explain the orange stripe in Figure 1 — see [3, 4, 5] for more details. In a supersymmetric theory, the kinetic mixing operator induces a mixing between the D-terms associated with $U(1)^{\prime}$ and $U(1)_{Y}$. The hypercharge D-term gets a vacuum expectation value from electroweak symmetry breaking and induces a weak-scale effective Fayet-Iliopoulos term for $U(1)^{\prime}$. Consequently, the Standard Model vacuum can break the $U(1)^{\prime}$ in the presence of light $U(1)^{\prime}$-charged degrees of freedom, giving the $A^{\prime}$ a mass,

$$
m_{A^{\prime}} \sim \sqrt{\epsilon g_{D}} \frac{\sqrt{g_{Y}} m_{W}}{g_{2}}
$$

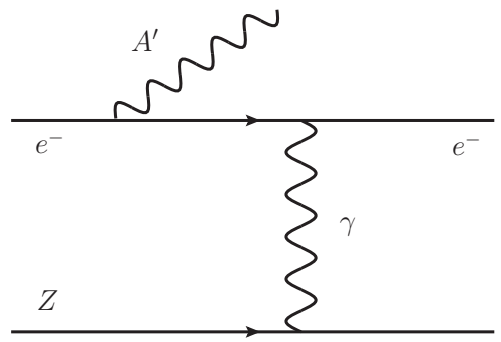

FIG. 2: $A^{\prime}$ production by bremsstrahlung off an incoming electron scattering off protons in a target with atomic number $Z$.

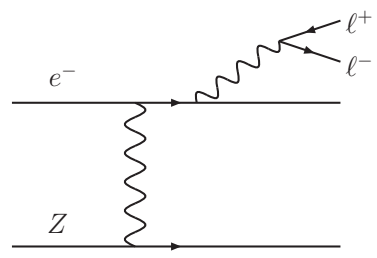

(a)

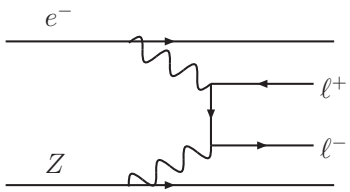

(b)
FIG. 3: (a) $\gamma^{*}$ and (b) Bethe-Heitler trident reactions that comprise the primary QED background to $A^{\prime} \rightarrow \ell^{+} \ell^{-}$search channels.

where $g_{D}, g_{Y}$, and $g_{2}$ are the the $U(1)^{\prime}, U(1)_{Y}$, and Standard Model $S U(2)_{L}$ gauge couplings, respectively, and $m_{W}$ is the $\mathrm{W}$-boson mass. Equation (4) relates $\epsilon$ and $m_{A^{\prime}}$ as indicated by the orange stripe in Figure 1 for $g_{D} \sim 0.1-1$. This region is not only theoretically appealing, but also roughly corresponds to the region in which the annual modulation signal observed by DAMA/LIBRA can be explained by dark matter, charged under the $U(1)^{\prime}$, scattering inelastically off nuclei through $A^{\prime}$ exchange. We therefore include these lines for reference in our plots.

\section{B. $A^{\prime}$ Production in Fixed-Target Collisions}

$A^{\prime}$ particles are generated in electron collisions on a fixed target by a process analogous to ordinary photon bremsstrahlung, see Figure 2. This can be reliably estimated in the Weizsäcker-Williams approximation (see Appendix A for more details) [38, 39, 40]. When the incoming electron has energy $E_{0}$, the differential cross-section to produce an $A^{\prime}$ of mass $m_{A^{\prime}}$ with energy $E_{A^{\prime}} \equiv x E_{0}$ is

$$
\begin{aligned}
& \frac{d \sigma}{d x d \cos \theta_{A^{\prime}}} \approx \frac{8 Z^{2} \alpha^{3} \epsilon^{2} E_{0}^{2} x}{U^{2}} \mathcal{L} o g \\
& \quad \times\left[\left(1-x+\frac{x^{2}}{2}\right)-\frac{x(1-x) m_{A^{\prime}}^{2}\left(E_{0}^{2} x \theta_{A^{\prime}}^{2}\right)}{U^{2}}\right]
\end{aligned}
$$

where $Z$ is the atomic number of the target atoms, $\alpha \simeq 1 / 137, \theta_{A^{\prime}}$ is the angle in the lab frame between the emitted $A^{\prime}$ and the incoming electron, the $\mathcal{L}$ og $(\sim 5-10$ 
for $\left.m_{A^{\prime}} \lesssim 500 \mathrm{MeV}\right)$ depends on kinematics, atomic screening, and nuclear size effects (see Appendix $\mathrm{A}$ and Figure 10 therein), and

$$
U\left(x, \theta_{A^{\prime}}\right)=E_{0}^{2} x \theta_{A^{\prime}}^{2}+m_{A^{\prime}}^{2} \frac{1-x}{x}+m_{e}^{2} x
$$

is the virtuality of the intermediate electron in initialstate bremsstrahlung. The above results are valid for

$$
m_{e} \ll m_{A^{\prime}} \ll E_{0}, \quad x \theta_{A^{\prime}}^{2} \ll 1 .
$$

Dropping $m_{e}$ and performing the angular integral, we find

$$
\frac{d \sigma}{d x} \approx \frac{8 Z^{2} \alpha^{3} \epsilon^{2} x}{m_{A^{\prime}}^{2}}\left(1+\frac{x^{2}}{3(1-x)}\right) \mathcal{L} o g
$$

The $x$-scaling and singularity structure $\frac{\alpha^{3}}{m_{e}^{2} x}$ of massless bremsstrahlung [40] is recovered from (5) with $m_{A^{\prime}}^{2}=0$ (the polynomial factor differs because of finite $m_{A^{\prime}}$ corrections to the matrix element), but differs from the massive $A^{\prime}$-strahlung in several important ways. We emphasize that these properties are not particular to any matrix element, but rather is a kinematic property common to all heavy-particle emission:

Rate: For most $x, U(x, 0) \sim m_{A^{\prime}}^{2}$, so that the total $A^{\prime}$ production rate is controlled by $\frac{\alpha^{3} \epsilon^{2}}{m_{A^{\prime}}^{2}}$. Therefore, it is suppressed relative to photon bremsstrahlung by $\sim \epsilon^{2} \frac{m_{e}^{2}}{m_{A^{\prime}}^{2}}$.

Energy: $A^{\prime}$ bremsstrahlung is sharply peaked at $x \approx$ 1 , where $U(x, 0)$ is minimized. When an $A^{\prime}$ is produced, it carries nearly the entire beam energy - in fact the median value of $(1-x)$ is $\sim \max \left(\frac{m_{e}}{m_{A^{\prime}}}, \frac{m_{A^{\prime}}}{E_{0}}\right)$.

Angle: $A^{\prime}$ emission is dominated at angles $\theta_{A^{\prime}}$ such that $U\left(x, \theta_{A^{\prime}}\right) \lesssim 2 U(x, 0)$ (beyond this point, wideangle emission falls as $1 / \theta_{A^{\prime}}^{4}$ ). For $x$ near its median value, the cutoff emission angle is

$$
\theta_{\mathrm{A}^{\prime} \max } \sim \max \left(\frac{\sqrt{m_{A^{\prime}} m_{e}}}{E_{0}}, \frac{m_{A^{\prime}}^{3 / 2}}{E_{0}^{3 / 2}}\right)
$$

which is parametrically smaller than the opening angle of the $A^{\prime}$ decay products, $\sim m_{A^{\prime}} / E_{0}$. The approximation of collinear emission is justified in many calculations.

Because these features apply to bremsstrahlung of any massive boson, there is a simple approximate equivalence between constraints on the $A^{\prime}$ and constraints on an axion with mass $m_{a}=m_{A^{\prime}}$ and decay constant $f_{a} \sim 1.7 \times 10^{-3} \mathrm{GeV} C_{e} / \epsilon$, at least when the constraints come from coupling to electrons. Here the coupling constant of axions to electrons is assumed to be $C_{e} m_{e} / f_{a}$, where $C_{e}$ is a model-dependent coefficient.
The total number of $A^{\prime}$ produced when $N_{e}$ electrons of initial energy $E_{0}$ scatter in a target of $T$ radiation lengths is

$$
\begin{aligned}
\frac{d N}{d x}=N_{e} \frac{N_{0} X_{0}}{A} & \int_{E_{A^{\prime}}}^{E_{0}} \frac{d E_{1}}{E_{1}} \int_{0}^{T} d t I\left(E_{1} ; E_{0}, t\right) \\
& \times\left. E_{0} \frac{d \sigma}{d x^{\prime}}\right|_{x^{\prime}=E_{A^{\prime}} / E_{1}}
\end{aligned}
$$

where $x^{\prime} \equiv E_{A^{\prime}} / E_{1}, X_{0}$ is the radiation length of the target, $N_{0} \simeq 6 \times 10^{23}$ mole $^{-1}$ is Avogadro's number, $A$ is the target atomic mass in $\mathrm{g} /$ mole, and $I$ is the energy distribution of electrons after passing through $t$ radiation lengths.

After the $A^{\prime}$ is produced in the target, it will travel for some distance before decaying back into Standard Model particles (we will assume throughout this paper that no other decay channels into particles charged under the $U(1)^{\prime}$ are available). The proper lifetime of the $A^{\prime}$ is

$$
\begin{aligned}
c \tau & =\frac{1}{\Gamma} \simeq \frac{3}{N_{\mathrm{eff}} m_{A^{\prime}} \alpha \epsilon^{2}} \\
& \simeq \frac{80 \mu \mathrm{m}}{N_{\mathrm{eff}}}\left(\frac{10^{-4}}{\epsilon}\right)^{2}\left(\frac{100 \mathrm{MeV}}{m_{A^{\prime}}}\right)
\end{aligned}
$$

where we have neglected phase-space corrections and $N_{\text {eff }}$ counts the number of available decay products $\left(N_{\text {eff }}=1\right.$ for $m_{A^{\prime}} \lesssim 2 m_{\mu}$ when only $A^{\prime} \rightarrow e^{+} e^{-}$decays are possible, and $2+R\left(m_{A^{\prime}}\right)$ for $m_{A^{\prime}} \geq 2 m_{\mu}$, where $R$ is defined to be the energy dependent ratio $\frac{\sigma\left(e^{+} e^{-} \rightarrow \text { hadrons }\right)}{\sigma\left(e^{+} e^{-} \rightarrow \mu^{+} \mu^{-}\right)}$[41]). $A^{\prime}$ decays will thus create displaced vertices behind the target. While $c \tau$ determines the typical impact parameter for these displaced tracks, their vertex displacements are controlled by (for the typical kinematics with $x \approx 1$ ),

$$
\begin{aligned}
\ell_{0} & \equiv \gamma c \tau \simeq \frac{3 E_{1}}{N_{\mathrm{eff}} m_{A^{\prime}}^{2} \alpha \epsilon^{2}} \\
& \simeq \frac{0.8 \mathrm{~cm}}{N_{\mathrm{eff}}}\left(\frac{E_{0}}{10 \mathrm{GeV}}\right)\left(\frac{10^{-4}}{\epsilon}\right)^{2}\left(\frac{100 \mathrm{MeV}}{m_{A^{\prime}}}\right)^{2}
\end{aligned}
$$

where we have again neglected phase-space corrections.

\section{Approximate Total Rate Formulas}

From equations (8) and 12 , we can obtain simple approximate expressions for the rate of $A^{\prime}$ production in scattering off thin targets (with $T \ll 1$ ) and thick "dump" targets $(T \gg 1)$. These crude approximations are only correct within about one order of magnitude, but they are useful in quickly mapping out regions in the large logarithmic parameter space. In our results we use more accurate expressions that also include detector acceptances, as presented in Appendices $\mathrm{A}$ and $\mathrm{B}$.

In the thin-target limit $T \ll 1$, the beam is not significantly degraded as it passes through the target, and 
$I\left(E_{1}, E_{0}, t\right) \approx \delta\left(E_{1}-E_{0}\right)$. In this case the total $A^{\prime}$ production rate scales as

$$
N \sim N_{e} \frac{N_{0} X_{0}}{A} T \frac{Z^{2} \alpha^{3} \epsilon^{2}}{m_{A^{\prime}}^{2}} \mathcal{L} o g=N_{e} \mathcal{C} T \epsilon^{2} \frac{m_{e}^{2}}{m_{A^{\prime}}^{2}},
$$

where $\mathcal{C} \approx 5$ is only logarithmically dependent on the choice of nucleus (at least in the range of masses where the form-factor is only slowly varying) and on $m_{A^{\prime}}$, because, roughly, $X_{0} \propto \frac{A}{Z^{2}}$ (see Appendix B and 41]). For example, for a Coulomb of incident electrons

$$
\frac{N}{\mathrm{C}} \sim 10^{6}\left(\frac{T}{0.1}\right)\left(\frac{\epsilon}{10^{-4}}\right)^{2}\left(\frac{100 \mathrm{MeV}}{m_{A^{\prime}}}\right)^{2} .
$$

For a thick target $(T \gg 1)$, production is dominated near the front of the target and

$$
N \sim N_{e} \mathcal{C}^{\prime} \epsilon^{2} \frac{m_{e}^{2}}{m_{A^{\prime}}^{2}},
$$

with $\mathcal{C}^{\prime} \approx 10$. When the typical lifetime $\ell_{0}$ exceeds the length $L$ to the detector, a fraction $\sim L / \ell_{0}$ decay before the detector, and the number of $A^{\prime}$ observed is independent of $m_{A^{\prime}}$ :

$$
N_{\text {obs }} \sim N_{e} \mathcal{C}^{\prime} \epsilon^{2} \frac{m_{e}^{2}}{m_{A^{\prime}}^{2}} \frac{L}{\ell_{0}} \sim N_{e} \mathcal{C}^{\prime} \alpha \epsilon^{4} \frac{m_{e}^{2} L}{E_{1}}
$$

Note that multiple interactions in the target degrade the beam energy significantly and induce $A^{\prime}$ transverse momenta $\sim 10 \mathrm{mrad}\left(\mathrm{GeV} / E_{0}\right)^{2}$ for $A^{\prime}$ production in the first radiation length. These transverse momenta can be significant for low-energy dumps.

For subsequent discussions, it is useful to translate the signal yields into rates as a function of beam and target parameters. A process $X$ with cross section $\sigma(X)$ occurs with a rate

$$
\Phi(X) \sim 0.7 \mathrm{MHz}\left[\frac{T \cdot I_{\text {beam }}}{\mathrm{nA}} \frac{\sigma(X)}{Z^{2} \mu \mathrm{b}}\right],
$$

where $I_{\text {beam }}$ is the average current, and $T \lesssim 1$ is the target thickness in units of radiation lengths. For example, $m_{A^{\prime}}=100 \mathrm{MeV}$ and $\epsilon=10^{-4}$ gives $\sigma_{A^{\prime}} \sim 0.01 Z^{2} \mathrm{pb}$, or a rate of $\Phi_{A^{\prime}} \approx 0.007 \mathrm{~Hz}\left[\frac{T \cdot I_{\text {beam }}}{\mathrm{nA}}\right]$. In contrast, BetheHeitler pair production (Figure 3) has a total crosssection $\sigma_{l^{+} l^{-}} \sim Z^{2} \mu \mathrm{b}$, or rate $\Phi_{l^{+} l^{-}} \approx 0.7 \mathrm{MHz}\left[\frac{T \cdot I_{\text {beam }}}{\mathrm{nA}}\right]$ for a $\sim 1 \mathrm{GeV}$ electron beam. Bethe-Heitler pair production is thus a potential background for any $A^{\prime}$ search, and so our experimental scenarios will be strongly influenced by the need to remove them.

In appendix C, we discuss the kinematics of BetheHeitler production relative to $A^{\prime}$ production in some detail, and sketch out a set of selection cuts that can be used to suppress the otherwise prohibitively large BetheHeitler backgrounds (Figure 3(b)). However, a minimal contribution to the background is obtained by replacing the $A^{\prime}$ by a $\gamma^{*}$ (Figure 3(a)). To see this, we re-insert the dilepton invariant mass $m^{2}$ into the fully differential cross-section and consider integrating over a mass window $\delta m$, with $\Gamma \ll \delta m \ll m$. The $A^{\prime}$ and $\gamma^{*}$ matrix elements are related by the substitution

$$
\frac{\epsilon^{2}}{m^{2}-\left(m_{A^{\prime}}^{2}+i m_{A^{\prime}} \Gamma\right)^{2}} \rightarrow \frac{1}{m^{2}} .
$$

All other terms in the cross-section are slowly varying in this window. Treating them as constant, the integration over the mass window bounded by $m \pm \frac{\delta m}{2}$ is straightforward. Substituting (11), we obtain the ratio of fully differential cross sections for $A^{\prime}$ to $\gamma^{*}$ production in this mass window, which is also an upper bound on the total signal to background,

$$
\frac{d \sigma\left(X \rightarrow A^{\prime} Y \rightarrow l^{+} l^{-} Y\right)}{d \sigma\left(X \rightarrow \gamma^{*} Y \rightarrow l^{+} l^{-} Y\right)}=\left(\frac{3 \pi \epsilon^{2}}{2 N_{f} \alpha}\right)\left(\frac{m_{A^{\prime}}}{\delta m}\right),
$$

where $N_{f}$ is the number of available decay species for the $A^{\prime}$, and $\delta m$ is the width assigned to the $\gamma^{*}$ process. Equation (19) summarizes the maximum achievable signal to background ratio that any experiment can achieve in an $A^{\prime} \rightarrow \ell^{+} \ell^{-}$search using only kinematics, with the decay vertex unresolved.

\section{BEAM DUMP CONSTRAINTS AND SENSITIVITY OF CURRENT EXPERIMENTS}

In this section, we discuss existing constraints on the $\epsilon$ versus $m_{A^{\prime}}$ parameter space, which are summarized in Figure 1 .

For $m_{A^{\prime}}>2 m_{\mu}$, a search for $\Upsilon(3 S) \rightarrow \gamma A^{\prime} \rightarrow \gamma \mu^{+} \mu^{-}$ by the BaBar collaboration [42] rules out $\epsilon \gtrsim 10^{-3}$ (see also [32]), while the electron and muon anomalous magnetic moments rule out the low-mass-high- $\epsilon$ region 43 .

Strong constraints are also obtained from electron beam-dump experiments searching for $\mathrm{MeV}$-mass axions. The strongest constraints come from the E137 [4] and E141 [45] experiments at SLAC, and the E774 [46] experiment at Fermilab:

SLAC E137 dumped $30 \mathrm{C}$ of electrons at $20 \mathrm{GeV}$ into aluminum targets 44. Beam products traveled through a $200 \mathrm{~m}$ hill and an additional $200 \mathrm{~m}$ of open region before hitting a $(3 \mathrm{~m})^{2}$ detector. No candidate events were observed. The contour in Figure 1 represents an expected signal of 10 events (we have idealized the detector as a circle of radius $1.5 \mathrm{~m})$.

SLAC E141 dumped $2 \times 10^{15}$ electrons at $9 \mathrm{GeV}$ into a 12-cm tungsten target, with a $10-\mathrm{cm}$ tungsten target used for calibration [45]. The detector was located $35 \mathrm{~m}$ from the dump, and the analysis required observing a single decay product carrying over 0.5 times the beam energy with angular acceptance set by a 7.5 -cm pipe. Based on the background rates reported by the experiment, the ex- 
clusion in Figure 1 represents an expected signal of 1000 events.

Fermilab E774 dumped $0.52 \times 10^{10}$ electrons at 275 $\mathrm{GeV}$ onto two 28-radiation-length-thick (about 19.6-cm) stacks of tungsten plates 46. The overall target length, including veto counters behind the target, was 30-cm. An electromagnetic calorimeter with an angular acceptance of about $20-\mathrm{cm}$ was placed $7.25 \mathrm{~m}$ downstream from the dump. The trigger required an energy deposition of at least $27.5 \mathrm{GeV}$ and no signal from the veto counters. Based on the results reported by the experiment, Figure 1 represents an expected signal of 17 events.

The approximate formulas given in Section II C are sufficient to understand the shape and magnitude of the beam-dump limits shown in Figure 1 they are bounded above by a diagonal along which many $A^{\prime}$ may be produced, but all decay within the shielding that stops the beam, and from below by a line of diminishing rate, which is diagonal if the typical decay occurs before the detector position, and approximately horizontal if the average decay length $\ell_{0}$ is larger than the length scale $L$ of the experiment (see equation 16)). Similar limits can be derived for alternative $A^{\prime}$ decay modes, for example if the $A^{\prime}$ decays to dark-sector higgses with typical proper lifetime that scales as $\epsilon^{-4}$ rather than $\epsilon^{-2}$.

Supernova cooling places a significant constraint on lower $\epsilon$ and lighter $m_{A^{\prime}}$. A proper accounting of supernova limits on the $A^{\prime}$ is beyond the scope of this paper, but we outline a simple estimate based on scaling similar results for axions [47. The hot core of a collapsing supernova can cool through production of $A^{\prime}$ if they decay $\gtrsim 10 \mathrm{~km}$ from the point of production (the mean free path is typically longer than the lifetime). However, neutrino observations of SN1987A confirmed an energy loss over $5-10$ seconds of $1-4 \times 10^{53} \mathrm{erg}$. Following [4], we require the energy loss in $A^{\prime}$ emission not to exceed $10^{53} \mathrm{erg} / \mathrm{s}$. We take the $A^{\prime}$ luminosity per unit energy from the core to be

$$
\frac{d L}{d E_{A}} \sim \frac{1}{T_{S N}}\left(6 \times 10^{70} \mathrm{erg} / \mathrm{s}\right) e^{2} \epsilon^{2}
$$

for $E_{A}<T_{S N}=30 \mathrm{MeV}$, which is suppressed by $T_{S N} / m_{p}$ (where $m_{p}$ is the proton mass) relative to the axion rate 47, 48 because the vector emission matrix element is proportional to $v^{2}$, whereas the axion emission matrix element approaches a constant as $v \rightarrow 0$. We impose an additional Boltzmann suppression $e^{-E_{A} / T}$ for $E_{A}>T_{S N}$ and multiply by the fraction $f\left(E_{A}\right)=$ $e^{-10 \mathrm{~km} / \ell_{0}}$ that leave the supernova core. Requiring that the total luminosity not exceed $10^{53} \mathrm{erg} / \mathrm{s}$, we can exclude the lower-most region in Figure 1. We emphasize that the luminosity obtained by scaling is only correct within an order of magnitude. An error in the crosssection would affect the lower limit in $\epsilon$ proportionally, but the upper limit only logarithmically.
Constraints from other experiments are all contained within the limits from the experiments discussed above. For example, the region constrained by the SLAC search for milli-charged particles, which also used an electron beam, is contained within E137 [49. Proton beam dumps can produce $A^{\prime}$ in radiation directly from the proton or in radiation from electrons produced by the nuclear shower. Both processes produce $A^{\prime}$ of much lower energy than the primary proton (hard bremsstrahlung off the proton is suppressed by the proton's finite size, and the shower electrons are quite soft). These softer $A^{\prime}$ typically decay inside the dump. Therefore, proton dumps such as the CHARM experiment at CERN [50] do not exclude new regions, though they do overlap significantly with the E137 exclusion. Likewise, experiments dumping proton beams for other purposes (e.g. neutrino experiments such as MINOS and MINIBOONE) have little or no potential reach beyond E137.

We have also considered potential limits from $A^{\prime}$ production off cosmic rays impinging on Super-K, AMANDA, and ICE-CUBE detectors, which is dominated by bremsstrahlung off muons near ground-level, which must only survive $\sim 1 \mathrm{~km}$ to reach the detectors. The potential sensitivities of these experiments are contained within the E137 excluded region.

\section{SCENARIOS FOR NEW EXPERIMENTS}

The parameter space that new experiments must cover spans a huge range. The $A^{\prime}$ production cross section and decay width vary as $\epsilon^{2}$ and thus vary over ten orders of magnitude. Our purpose in this section is to explore experimental scenarios appropriate to different parameter ranges. For the sake of definiteness, we organize the discussion around parameter points labeled "A" through "E" in Figure 1, Each choice suggests a different experimental approach, described in the appropriately labeled subsections.

We do not intend here to provide detailed designs; this is the task of those who would actually do the experiments. We do attempt to show that the exploration of this parameter space is experimentally feasible and offer some guidance regarding how to choose design parameters in order to optimize the experimental sensitivity. Because the electron beams at Jefferson Laboratory appear to be an attractive choice for such experiments, we have been guided in our considerations by the beam specifications available there. However, we expect that other attractive options exist elsewhere.

There is a natural dividing line in the parameter space (cf. Figure 1), corresponding to an $A^{\prime}$ proper lifetime $c \tau \simeq 80 \mu \mathrm{m}$, comparable to that of the $\tau$ lepton. Longer lifetimes allow in principle the determination of a separated decay vertex, while much shorter lifetimes do not. Since determination of the detached vertex is a strong experimental signature, the experimental techniques naturally differ in the two regimes. Beam-dump searches, 
including our first (and last) scenario, are appropriate to much longer lifetimes. The region near the dividing line has not yet been explored, and micro-vertex detectors appear quite promising in this range. The second and third scenarios we describe assume this technique.

For very short lifetimes of the $A^{\prime}$, the experimental signature is identical to electromagnetic trident production $e+Z \rightarrow 3 e$ or $e+Z \rightarrow e+2 \mu$ (where $Z$ is the target), shown in Figure 3 The simple upper bound discussed in equation $\sqrt{19}$ in Section II on the ratio of the $A^{\prime}$ fully differential cross-section to the background trident rate implies that high statistics and resolution are required to have any chance of observing the $A^{\prime}$. Fortunately, such a regime does appear to overlap with the capabilities of Jefferson Laboratory spectrometers. Moreover, the upper bound is attained in a sizeable region of the differential phase space where $A^{\prime}$ production is dominant, if appropriate kinematic cuts are applied on the final state leptons (see Appendix C). Therefore this parameter region seems in principle to be accessible. Our fourth experimental scenario deals with this case. We will also show that the second and third scenarios we discuss have some interesting new reach in parameter space when used at lower luminosity as high-resolution forward spectrometers.

For the smallest values of $\epsilon$, the primary consideration is simply producing enough $A^{\prime}$ s to study experimentally, so beam-dump experiments are the technique of choice. However, it becomes very challenging to design beam dumps with average power exceeding 1 megawatt (MW). There appears to be a small window of opportunity available for such a search, which would increase the reach beyond that of E137. This comprises the fifth scenario that we discuss.

\section{A. Low Power, $10 \mathrm{~cm}$ Tungsten Beam Dump; $\epsilon=10^{-5} ; m_{A^{\prime}}=50 \mathrm{MeV}$}

We consider a $200 \mathrm{MeV}$ primary electron beam incident on a $10 \mathrm{~cm}$ tungsten target. Downstream (beyond a thin, but dense shielding wall, if necessary), is an instrumented decay volume containing a combination of tracking planes, electromagnetic calorimetry, and scintillator triggers. With the chosen values of $\epsilon$ and $m_{A^{\prime}}$, the laboratory decay length of a typical $A^{\prime}$ of momentum 160 $\mathrm{MeV}$ is about $5 \mathrm{~cm}$. The produced $A^{\prime}$ s are contained in an angular cone of order $125 \mathrm{mrad}$. Therefore the tracking system in an experimental region no more than 40 $\mathrm{cm}$ (see Figure 4) downstream of the front of the dump need have transverse dimensions no more than $10 \mathrm{~cm}$ to identify the $A^{\prime}$ decay vertices and measure the angles. Since the decay angles of the electron/positron pair are of order $250 \mathrm{mrad}$, the calorimeter transverse dimensions can be very modest.

In this scenario, the total yield per incident electron of $A^{\prime}$ s containing at least 80 percent of the beam momentum is about $9 \times 10^{-15}$ per electron dumped. If the front of the fiducial decay volume can be located immediately behind the $10 \mathrm{~cm}$ target, $5 \%$ of these $A^{\prime}$ s decay outside the target for a rate of $4 \times 10^{-16}$ per electron dumped. If a thicker shield is necessary to stop soft photons, the yield remains large: $0.1 \%$ of the $A^{\prime}$ decay beyond $30 \mathrm{~cm}$ from the front of the dump, for one observable $A^{\prime}$ decay per $5 \times 10^{16}$ electrons dumped. In the conservative configuration, with a total of $30 \mathrm{~cm}$ of material, a yield of order 30 events would be observed for 0.3 coulombs of electrons dumped $(300 \mathrm{nA}$ in an experiment of duration $10^{6}$ seconds). This requires a modest 60 watts of beam power on the tungsten dump.

The length of the fiducial decay region need only be 20 centimeters to capture the majority of the $A^{\prime}$ s emerging from the dump into the decay region. The compact nature of this decay volume suggests the possible use of silicon strip detectors for the tracking system.

The question of backgrounds must of course be addressed. A fast, dense tracking system seems to be appropriate. With a readout rate at least $10 \mathrm{MHz}$, and with a continuous incident beam such as exists at Jefferson Laboratory, there would be about 30000 electrons dumped per readout cycle. The shower products should be absorbed efficiently, and it should be very rare that a prompt calorimeter signal of more than $100 \mathrm{MeV}$ energy deposition occurs, especially because the time resolution of a scintillator trigger/electromagnetic calorimeter system will be much better than $100 \mathrm{~ns}$. The residual problems, beyond the scope of this sketch, probably have to do with neutrons and soft photons or x-rays.

Evidently, if we lower $m_{A^{\prime}}$ and increase $\epsilon$ in such a way as to decrease neither the rest-frame decay length nor the production rate, the experiment will be easier, since $\gamma c \tau$ is larger and more $A^{\prime}$ will decay in the detector volume. In Figure 4 we present our rough estimate of the region of parameter space accessible to the experiment as described.

To extend the reach to larger masses and smaller $\epsilon$, higher beam energies are required. Once the threshold for electro-production of muons and hadrons has been crossed, the experiment may require a thicker shield, and much higher energies appear advantageous. For example, consider raising $m_{A^{\prime}}$ and lowering $\epsilon$ by a factor of 2 , to $100 \mathrm{MeV}$ and $5 \times 10^{-6}$. For a beam energy of $6 \mathrm{GeV}$, the decay length is $2 \mathrm{~m}$. If a $3 \mathrm{~m}$ decay volume can be positioned with its upstream end within $4 \mathrm{~m}$ of the front of the dump, then a larger fraction of the produced $A^{\prime}$ s can be detected than our original example. This in turn lessens the beam-intensity requirement; an average current of $100 \mathrm{nA}$, which leads to a dump power of under 1 kilowatt, appears to suffice.

The detector geometry can simply be a longitudinally stretched version of the previous case, with transverse dimensions again quite small, of order $15-20 \mathrm{~cm}$. However, new backgrounds appear. Muons will penetrate the decay volume as well as electromagnetic showers initiated within the hadronic cascade. One leading candidate for background troubles comes from electro-production 

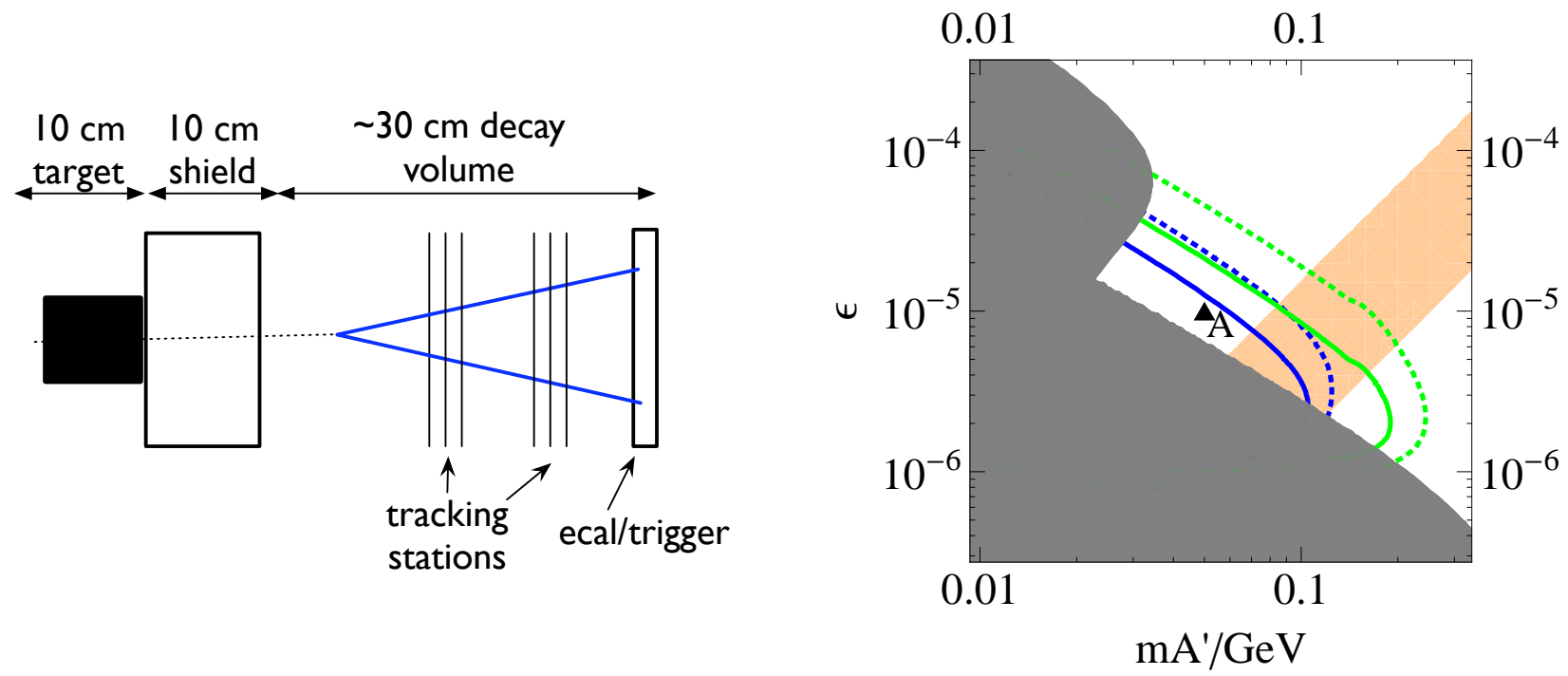

FIG. 4: Left: Experimental scenario for benchmark point $\mathrm{A}\left(\epsilon \sim 10^{-5}, m_{A^{\prime}} \sim 50 \mathrm{MeV}\right)$. An electron beam is incident on a 10 $\mathrm{cm}$ thick tungsten target. Behind the target is a $10 \mathrm{~cm}$ (or thicker) shield followed by an instrumented decay region consisting of a combination of tracking planes, electromagnetic calorimetry and scintillator triggers. Right: Reaches of the high- and low-energy dump configurations described in Section IV A delineated by regions with 10 or more events and the following configurations - Blue (inner) Solid Contour: $0.3 \mathrm{C}$ total charge dumped with a $200 \mathrm{MeV}$ electron beam, a $20 \mathrm{~cm}$ shield, and a detector with $5 \mathrm{~cm}$ radius $50 \mathrm{~cm}$ behind the front of the target. The lepton pair must have total energy exceeding $100 \mathrm{MeV}$. Blue (inner) Dashed Contour: same configuration, but with no shield. Green (outer) Solid Contour: $0.1 \mathrm{C}(100 \mathrm{nA}$ beam $\times$ $10^{6} \mathrm{~s}$ ) total charge dumped with a $6 \mathrm{GeV}$ electron beam, a $3.9 \mathrm{~m}$ shield, and a detector with $10 \mathrm{~cm}$ radius $7 \mathrm{~m}$ downstream. The lepton pair must have total energy exceeding $3 \mathrm{GeV}$. Green (outer) Dashed Contour: same configuration, but with $0.9 \mathrm{~m}$ of shielding. Gray contours and Orange Stripe: exclusions from past experiments (E137 and E141) and the region that explains DAMA/LIBRA in a simple model — see Figure 1 for more details.

of the $\rho$, with a leading charged pion from the rho decay undergoing a charge-exchange reaction into a $\pi^{0}$ a few radiation lengths in front of the detector region. We have used the experience obtained in E141 to make rough estimates, which indicate that such backgrounds are surmountable. But the soft backgrounds such as neutrons and hard x-rays also need to be carefully studied.

\section{B. Thin Target and Double Arm Spectrometer; $\epsilon=3 \times 10^{-5} ; m_{A^{\prime}}=200 \mathrm{MeV}$}

Modern micro-vertex detectors allow much better lifetime resolution than the above example. When $\epsilon$ is increased from the previous example, the rate of $A^{\prime}$ production per incident electron increases, and a thin target can be used instead of a beam dump. For the parameters of interest here, we consider a 0.1 radiation length tungsten target. We choose a $6 \mathrm{GeV}$ beam with an average current of $100 \mathrm{nA}$. Downstream of the target is a two-arm mini-spectrometer with silicon strip detectors as the tracking elements, backed up with fast calorimeter/scintillator triggers.

With these parameters, the $A^{\prime}$ production rate (before acceptance) out of the target is about 10 per hour. The angular divergence of the $A^{\prime}$ beam is only about $5 \mathrm{mrad}$. The laboratory decay length is about $1 \mathrm{~cm}$, and the decay products of the $A^{\prime}$ have an average angle of about
35 mrad from the beam axis. A spectrometer with polar angle coverage of 20 to $55 \mathrm{mrad}$ and $50 \%$ azimuthal angle coverage has about $25 \%$ acceptance for the $A^{\prime}$ decay products. The trigger requirement includes the demand that the energies in each of the calorimeters are between 1 and $5 \mathrm{GeV}$, with the sum between 5 and $6 \mathrm{GeV}$. The tracking system must identify one track in each arm that points to the calorimeter hit (if the calorimeter is segmented) and is consistent with a decay-vertex origin. After reconstruction, additional kinematic constraints provide rejection power. In Figure 5, we show the reach of this experimental scenario for various geometries and different beam currents.

A major background is simultaneous elastic coulomb scattering in each arm. An elastically scattered electron deposits $6 \mathrm{GeV}$ in the calorimeter, and is rejected, but the singles rate must be below one per timing window (100 MHz or less for fast calorimeters). This requirement is safely met by the beam intensity quoted above. The elastic-scattering radiative tails will contribute to the trigger, but at a significantly lower rate of $10 \mathrm{kHz}$ or so. Other sources for background triggers, such as BetheHeitler pair production (cf. Figure 3), lead to smaller or comparable trigger rates. When one of the two scattered electrons scatters again in the first layer of silicon, the intersection of the two reconstructed tracks is displaced. We find that the rate for these fake vertices is adequately suppressed if the first layer is placed close to the target, 

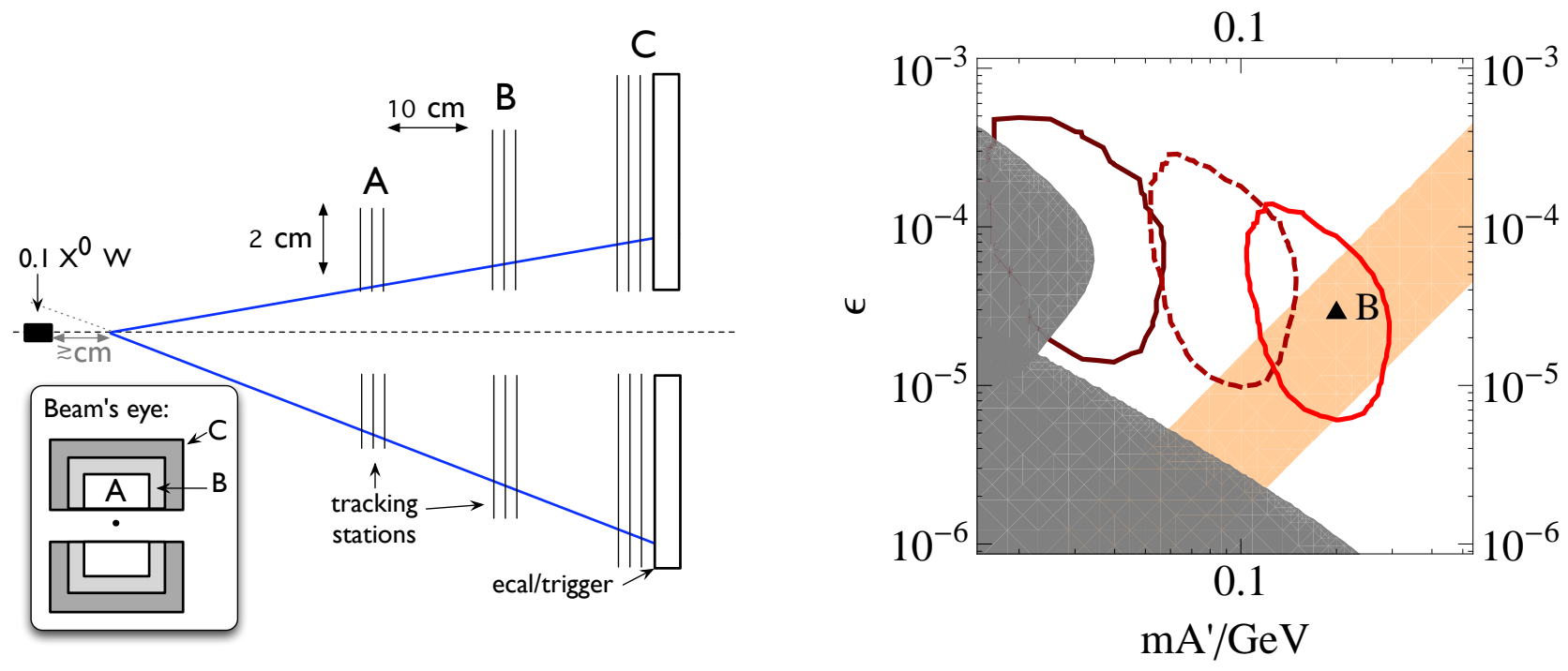

FIG. 5: Left: Experimental scenario for a small two-arm spectrometer for benchmark point B $\left(\epsilon \sim 3 \times 10^{-5}, m_{A^{\prime}} \sim 200\right.$ $\mathrm{MeV})$. An electron beam is incident upon a thin 0.1 radiation length tungsten target. A small two-arm spectrometer with silicon-strip trackers and a fast calorimeter or scintillator trigger is downstream from the target. Signal events are identified by requiring a displaced vertex $\sim 1 \mathrm{~cm}$ behind the target. More details are given in the text. Right: Regions corresponding to 10 or more events within acceptance in $10^{6} \mathrm{sec}$ for three different geometries. From right to left: $6 \mathrm{GeV}$ electron beam at $100 \mathrm{nA}$ (0.1 C delivered), with angular acceptance from 20 to $55 \mathrm{mrad}$ and a $1 \mathrm{~m}$ long detector (solid red line); $6 \mathrm{GeV}$ beam at $5 \mathrm{nA}$ $\left(5 \times 10^{-3} \mathrm{C}\right.$ delivered), with angular acceptance from 10 to $27 \mathrm{mrad}$ in a $2 \mathrm{~m}$-long detector region (dashed darker red line $)$; and $2 \mathrm{GeV}$ beam at $0.5 \mathrm{nA}\left(5 \times 10^{-4} \mathrm{C}\right.$ delivered) with the same geometry as the dashed red line (solid dark red line). In all cases, we require that the $A^{\prime}$ carry at least $83 \%$ of the beam energy, the track impact parameters at the target exceed $50 \mu$ m, and the reconstructed vertex displacement exceed $1 \mathrm{~cm}$. We assume $50 \% \phi$ coverage. Gray contours and Orange Stripe: exclusions from past experiments (E137 and E141) and the region that explains DAMA/LIBRA in a simple model — see Figure 1 for more details.

within $\sim 5-10 \mathrm{~cm}$.

Another basic requirement is that the occupancy in the tracking system be acceptably low. High-resolution silicon strip detectors are beneficial in this regard. Within a cone of opening angle of $10 \mathrm{mrad}$ at a distance of 50 $\mathrm{cm}$ downstream of the target, we estimate that the density of electrons and photons produced in the target with energy above $1 \mathrm{MeV}$ is of order $10^{9} / \mathrm{cm}^{2} / \mathrm{s}$ [58]. In this scenario, the silicon is placed further from the beam, but this rate serves as a rough upper bound, which would give one percent occupancy for a $1 \mathrm{~cm} \times 25 \mu \mathrm{m}$ strip. While these numbers are encouraging, a serious simulation is certainly required.

\section{Silicon Strip Layers in a Diffuse Electron Beam; $\epsilon=10^{-4} ; m_{A^{\prime}}=50 \mathrm{MeV}$}

At even higher $\epsilon$ and lower masses, there exists the option of halving the number of silicon strip tracking elements and placing them directly into a defocused primary electron beam of low intensity. For this study, we choose the beam size to be about $1 \mathrm{~cm} \times 1 \mathrm{~cm}$ and the beam energy to be $1 \mathrm{GeV}$. The beam intensity is limited by silicon occupancy to about $10^{8} \mathrm{e}^{-} / \mathrm{s}$, if we require occupancy of about $1 \%$ in $1 \mathrm{~cm} \times 25 \mu \mathrm{m}$ strips with a timing window of $20-50$ ns.
Triggering is again accomplished by a calorimeter, with a strategy similar to case B and the same limitations. For $A^{\prime}$ masses of $20-50 \mathrm{MeV}$, decay opening angles $\sim 20-50$ mrad are anticipated, so the calorimeter must extend close to the beam. For simplicity we consider an annular calorimeter with angular coverage above $20 \mathrm{mrad}$ (for example, located at 2.5 meters from the target, with inner radius of $5 \mathrm{~cm}$ ). The beam electrons emerge from a 0.1 radiation-length tungsten target in a Molière distribution, with typical transverse momenta of $5 \mathrm{MeV}$. Therefore less than $1 \%$ of the electron beam hits the calorimeter, leading to a $\lesssim 1 \mathrm{MHz}$ singles rate, which is high but manageable for a trigger requiring two hits.

With these parameters the $A^{\prime}$ production rate is about 1 every ten hours. Off-line track reconstruction can be used to remove the backgrounds associated with the Coulomb scattering pile-up and other background sources, in particular Bethe-Heitler pair production from the target. The quality of the experiment will depend crucially on the precision of the vertex reconstruction using the silicon strip information. Our sample point has typical impact parameter $\sim 160 \mu \mathrm{m}$ and laboratory decay lengths of order $2.3 \mathrm{~mm}$, which should be cleanly resolvable. The sensitivity of this configuration, assuming several different resolutions, is illustrated in Figure 6.

For smaller masses, the calorimeter must be placed at 

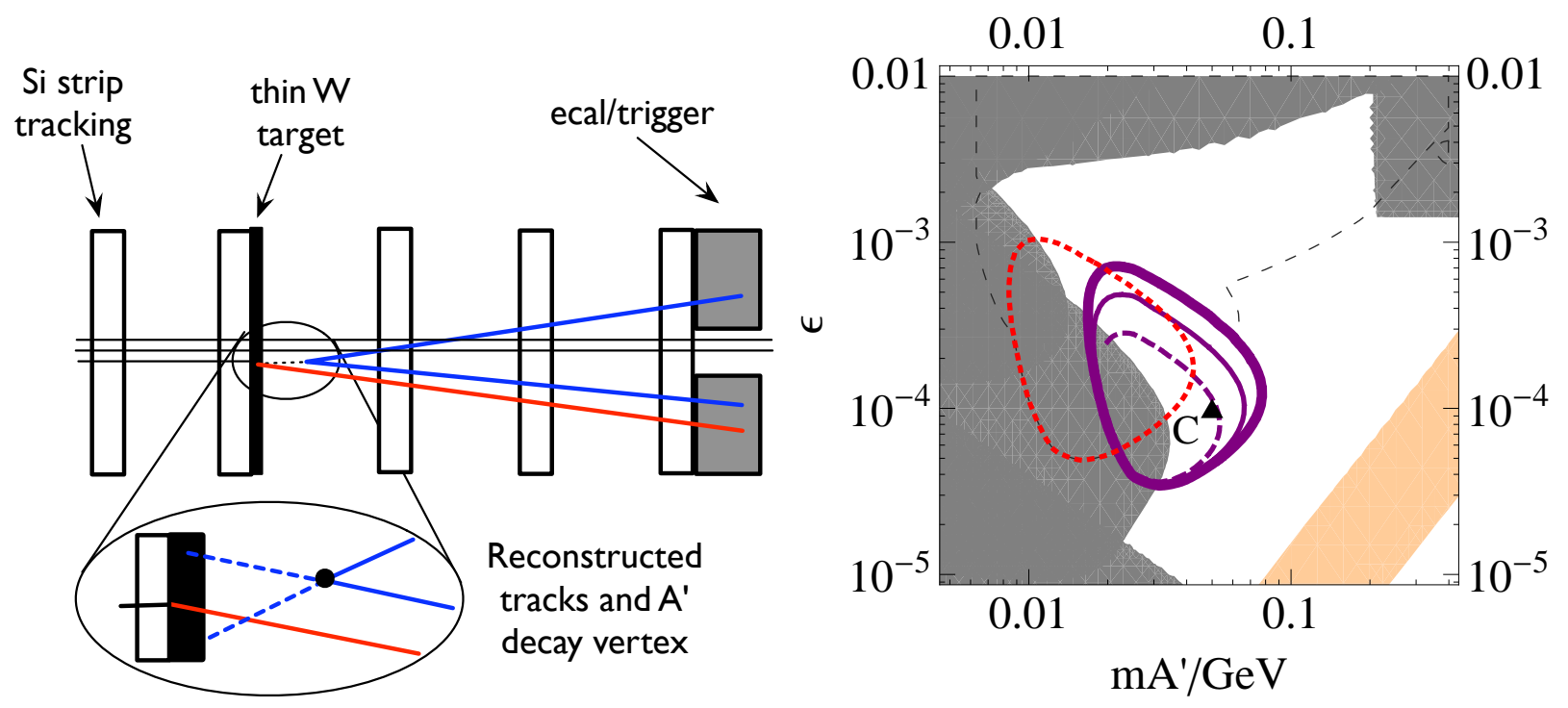

FIG. 6: Left: Experimental scenario for benchmark point $\mathrm{C}\left(\epsilon \sim 10^{-4}, m_{A^{\prime}} \sim 50 \mathrm{MeV}\right)$. Silicon strip tracking elements, together with a 0.1 radiation length $(300 \mu \mathrm{m})$ tungsten target directly behind one of the elements, are inserted into a $1 \mathrm{GeV}$ diffuse $(1 \mathrm{~cm} \times 1 \mathrm{~cm})$ electron beam of intensity $\lesssim 10^{8} \mathrm{e}^{-} / \mathrm{s}$. Triggering is accomplished by an annular calorimeter with angular coverage above $20 \mathrm{mrad}$ (e.g. $2 \mathrm{~cm}$ inner radius, $1 \mathrm{~m}$ downstream) by demanding three coincident hits carrying the beam energy. Signal events give rise to measurable impact parameters for the leading two tracks, and the excellent tracking provided by this design exploits this feature to reject background. Invariant mass reconstruction can provide an additional search variable (see Sec. IV D). More details are given in the text. Right: Concentric purple contours: Regions with detectable signal yield $\geq 10$ events, background rejection of $\sim 10^{-6}$ (yielding $S / B \gtrsim 1$ ), and an impact parameter of at least $33 \mu \mathrm{m}, 66 \mu \mathrm{m}$, or $150 \mu \mathrm{m}$, respectively, for the contours from the outside in. We assume a run time of $10^{6} \mathrm{~s}$ at $10^{8} \mathrm{e}^{-} / \mathrm{s}$. Red Dotted Contour: Analogous sensitivity with lower average current $\left(10^{7} \mathrm{e}^{-} / \mathrm{s}\right)$ and a smaller calorimeter aperture (10 mrad). Thin black dashed line: a rough estimate of the total region of sensitivity that could be accessible to this geometry using both displaced-vertex discrimination and invariant mass search windows with good momentum resolution (see Sec IV D). Gray contours and Orange Stripe: exclusions from past experiments (E137, E141, E774, electron and muon anomalous magnetic moments, and $\Upsilon(3 S)$ resonance searches) and the region that explains DAMA/LIBRA in a simple model — see Figure 1 for more details.

a narrower angle or the beam energy reduced. In either case, the Molière scattering becomes more acute. On the tails of the Molière distribution, one can compensate by lowering the intensity of the beam. At low beam intensities, a fast scintillator/calorimeter trigger system will resolve the passage of individual electrons in the beam (in a CW machine like CEBAF). Therefore, if the scintillator/calorimeter system is segmented (e.g. scintillating fiber calorimetry), the trigger requirement can be simultaneous deposition of the beam energy in more than one detection element - typically three. For larger masses, the beam intensity would have to be increased, and the silicon-strip occupancy presents a sharp barrier.

\section{High Resolution, High Rate Trident Spectrometer: $\epsilon=3 \times 10^{-4} ; m_{A^{\prime}}=1 \mathbf{G e V}$}

Large $A^{\prime}$ masses present two challenges: a low production rate and short $A^{\prime}$ lifetime. In the absence of a displaced vertex, the $A^{\prime}$ can only be observed as a small peak on the electromagnetic trident background. Reducing these backgrounds as much as possible is essential here. Additionally, targets with somewhat lower $Z$ than tung- sten are preferable in this high $A^{\prime}$ mass range in order to maintain charge coherence in scattering. For definiteness, we shall discuss the di-muon final state, though it is arguable that the electron-positron final state is preferable.

As discussed in Section II, the trident background arises from two subprocesses, which we call radiative and Bethe-Heitler (c.f. Figure 3). The radiative process gives an upper bound on the ratio of signal to background as in equation (19). The Bethe-Heitler process has a much larger $(\sim 100 \times)$ cross-section than the radiative trident process due to collinear logarithmic enhancements in the $e \rightarrow e \gamma$ splitting and sub-process $\gamma \gamma \rightarrow \mu \mu$. These enhancements can be avoided by demanding kinematically symmetric $\mu \mu$ decay products carrying the majority of the beam energy, and by demanding that the recoiling electron (if it can be identified) scatter at a wide angle. This preserves the large logarithm in the forward-peaked $A^{\prime}$ production cross-section, while regulating all logs in the Bethe-Heitler process. These selections are discussed further in Appendix C.

In addition to the trident processes, radiation of real photons by incident electrons, and their subsequent conversion in the target must be considered. This process 

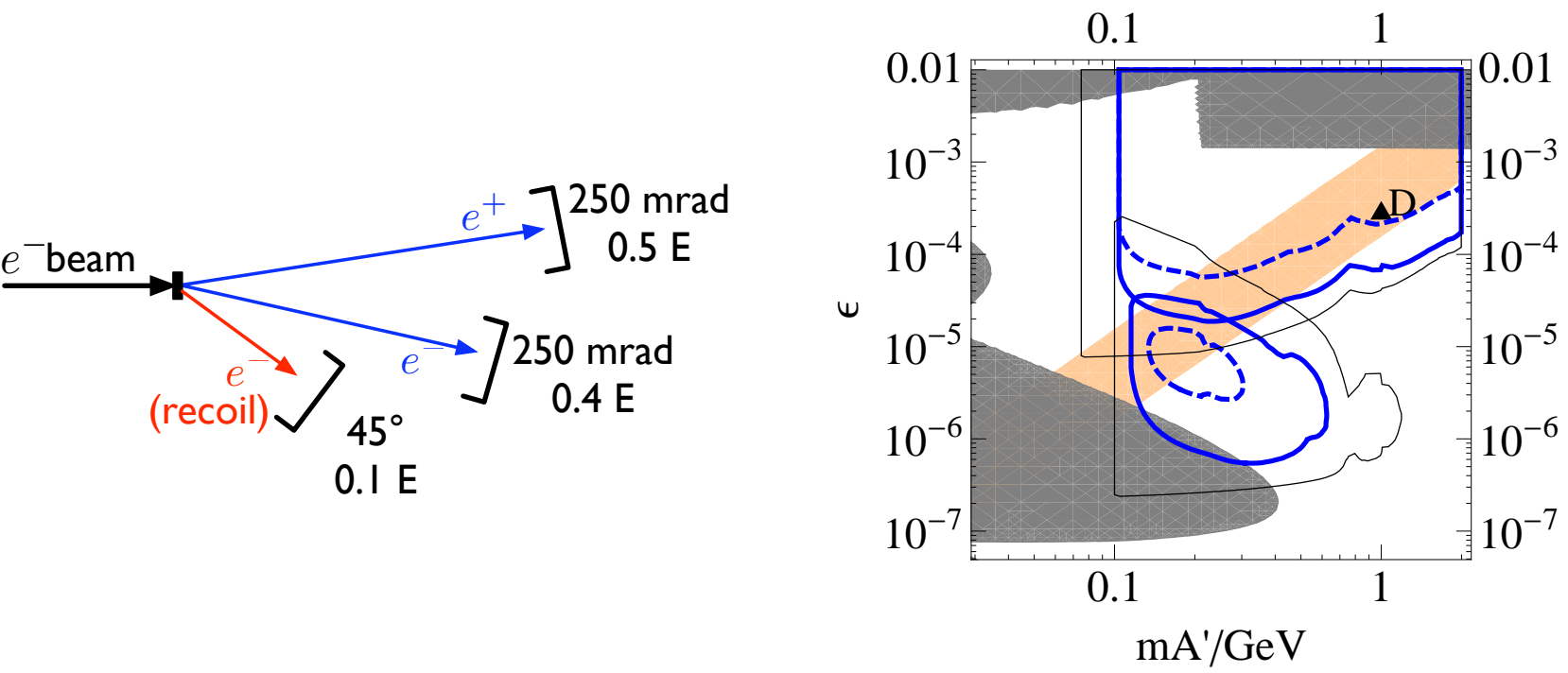

FIG. 7: Left: Schematic diagram of an experimental scenario for benchmark point D $\left(\epsilon \sim 3 \times 10^{-4}, m_{A^{\prime}} \sim 1 \mathrm{GeV}\right)$. An electron beam with an energy of $\sim 6 \mathrm{GeV}$ and a current of about $100 \mu \mathrm{A}-200 \mu \mathrm{A}$ is incident upon a 0.1 radiation length aluminum target. A wide-angle high-resolution spectrometer allows triggering on events in which one electron and one positron carry most of the beam energy. The signal is distinguished from background events with the help of various kinematic selection cuts (relatively symmetric $l^{+} l^{-}$final state and possible recoil electron tagging) and a "bump hunt" — see text and appendix C for further details. Right: Various estimates of the possible reaches of a wide-angle spectrometer, with (bottom) and without (top) tagging vertices displaced by $>1 \mathrm{~cm}$ to reject background. In each case, the outer thin black line represents a significant total rate, with no geometric acceptance requirements $(S / \sqrt{B}>5$ in the no-vertex (top) region, 10 or more events in the vertex (bottom) region). The thick blue curve shows the reach when decays are required to land more than 200 mrad away from the beam line, and the inner dotted curves assume an additional 1\% signal efficiency from acceptance. In these two cases, each curve represents the total reach obtained by running at several beam energies. Gray contours and Orange Stripe: exclusions from past experiments (E137, E141, E774, electron and muon anomalous magnetic moments, and $\Upsilon(3 S)$ resonance searches) and the region that explains DAMA/LIBRA in a simple model — see Figure 1 for more details.

is naively enhanced by $\mathcal{O}(T / \alpha)$ relative to Bethe-Heitler trident production, but can be rejected effectively with the same kinematic cuts. It is, of course, reducible by thinning the target, which allows a compensating increase in average beam current. We have not considered pile-up processes, but assume they are small when the three products are required to reproduce the beam energy within resolution.

For this scenario, we consider a 0.1-radiation-length aluminum target in a $4 \mathrm{GeV}$ beam. The total yield of $A^{\prime}$ s is roughly $10^{-16}$ per incident electron. If we assume an average beam current of $250 \mu \mathrm{A}$ (beam power of $1 \mathrm{MW}$ ) and an experimental duration of $10^{6} \mathrm{sec}$, the total rate of $A^{\prime}$ production is of order one per second, or $\gtrsim 10^{5}$ per experiment. These are emitted in a cone of size $\sim 100$ mrad, with decay products at opening angles near 250 mrad and the recoiling electron at a rather wide angle, 0.5 radians. The yield of background tridents having a di-muon mass within one percent of the $A^{\prime}$ mass is, according to 19 , about 300 times larger, or $3 \times 10^{7}$ per experiment. The estimated cumulative sensitivity of this configuration, and similar ones obtained by lowering the beam energy down to $\sim 1 \mathrm{GeV}$, is illustrated in Figure 7. To obtain the contours in this figure, we require that $S / \sqrt{B} \geq 5$, i.e. $\left(S / \epsilon_{b} B_{0}\right) \times S \geq 25$, where $S$ is the signal rate, and $B$ is the background rate, $B_{0}$, times the background rejection efficiency $\epsilon_{b}$. We use equation (19) to obtain $S / B_{0}$, and choose reasonable values for $\epsilon_{b}$.

The signal rate above is, indeed, larger than necessary for the $A^{\prime}$ resonance to be statistically significant. A less ambitious (and perhaps more realistic) experiment would also suffice for discovery. There are at least three ways to back off from this scenario. One way is evidently to improve the mass resolution. A second way is to reduce the beam intensity, keeping the acceptance complete. A reduction in beam current by a factor of 100 would still leave a viable signal. The third way is to reduce the acceptance; a one percent acceptance by itself would again leave a viable signal.

Optimization involves a choice of a combination of these factors. Jefferson Laboratory looks like an especially appropriate venue for this scenario, with two spectrometers with very good electron momentum resolution. In particular, the small-acceptance, high-rate spectrometers in Hall A has momentum resolution of order $10^{-4}$ and the large-acceptance Hall B CLAS detector has electron momentum resolution better than $1 \%$ [51. Therefore it would seem that using an electron-positron pair for the $A^{\prime}$ decay products may make more sense than using a di-muon pair. However, we feel further investigation is best done with the aid of expertise within the Jefferson Laboratory experimental community. 


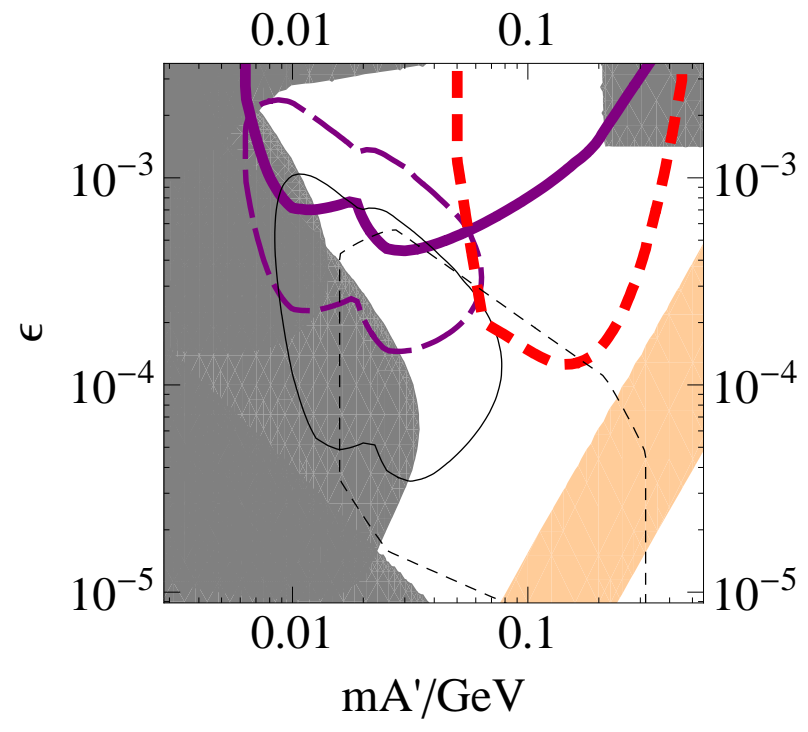

FIG. 8: Estimated reach for the geometries of scenarios B (thick dashed red) and $\mathrm{C}$ (thick solid and long-dashed purple) in "bump-hunt" operation with upgraded mass resolution. For reference, the approximate expected reach of clean vertex-based searches discussed in sections $\mathrm{B}$ and $\mathrm{C}$ is given by the thin, black dashed contour and the thin black solid contour, respectively. Thick Dashed Red Contour: The combined reach (with $S / \sqrt{B} \geq 5$ ) of the two two-arm spectrometer geometries introduced in Section IV B and Figure 5 with a $6 \mathrm{GeV}$ beam. Thick Solid Purple Contour: $S / \sqrt{B} \geq 5$ for the combined reach of the different "diffuse beam" scenarios in Section IV C assuming 1\% mass resolution. Long Dashed, Thinner Purple Contour: Same as the thick solid purple contour, but using vertexing to reduce backgrounds, with an assumed "optimistic" rejection of $10^{-2}$ for an impact parameter cut of $10 \mu \mathrm{m}$ applied to the lepton pair. Gray contours and Orange Stripe: exclusions from past experiments (E137, E141, E774, electron and muon anomalous magnetic moments, and $\Upsilon(3 S)$ resonance searches) and the region that explains DAMA/LIBRA in a simple model — see Figure 1 for more details.

\section{High-Resolution Forward Spectrometers at Lower Luminosity}

As we lower $m_{A^{\prime}}$ while keeping $\epsilon$ large, the production angles may become too low for big spectrometers such as those found in Hall A at JLab. The geometries of the detectors sketched in Sections IV B and IV C may be more appropriate. The growth of the production cross-section as $1 / m_{A^{\prime}}^{2}$ compensates for the lower average currents demanded by these scenarios (10-100 nA for the two-arm spectrometer B (described in Section IV B) and $10^{8} \mathrm{e}^{-} / \mathrm{s}$ when tracking planes are inserted in the beam as in $\mathrm{C}$ (described in Section IV C).

In scenarios $\mathrm{B}$ and $\mathrm{C}$, in the interest of simplicity, we did not assume a high mass resolution for the spectrometers. However, a high mass resolution, e.g. via addition of magnetic fields, is not at all impractical in principle. Assuming a $1 \%$ mass resolution, the potential sensitiv- ity of these modified scenarios is sketched in Figure 8. Again, it will be important to detect the wider-angle recoil electron in order to reduce backgrounds. This requires increasing the tracking and calorimeter coverage to around $250 \mathrm{mrad}$ for the two-arm spectrometer B as well as for the diffuse-beam, collinear configuration C.

The gap between the spectrometer and resolved vertex regimes coincides with $A^{\prime}$ decay lengths of order the $1 \sigma$ vertex resolution of a given detector. In this regime, one can gain sensitivity by imposing a loose impact parameter/vertex requirement that reduces background rates by a few orders of magnitude. Clearly, this strategy is plagued by the difficulties of both the spectrometry and vertexing approaches, and would only be attempted in a later stage when the detector and backgrounds are well understood. We do not discuss it further.

\section{E. High power, Low Energy Beam Dump; $\epsilon=5 \times 10^{-8} ; m_{A^{\prime}}=50 \mathrm{MeV}$}

Values of $\epsilon$ below the E137 limit require very intense beams simply to produce enough $A^{\prime}$ s to detect. Beam power limitations force one downward in beam energy. We choose a $200 \mathrm{MeV}$ beam of electrons with an average current of $5 \mathrm{~mA}$, representing a beam power of $1 \mathrm{MW}$. The $A^{\prime}$ production rate is about $2 \times 10^{-19}$ per electron dumped. The laboratory decay length is about $2.5 \mathrm{~km}$. The divergence of the $A^{\prime}$ beam is about $100 \mathrm{mrad}$. We consider a decay region $5 \mathrm{~m}$ long, with its front end located 5 meters downstream of the dump. A tracking system, perhaps in the style of E137, with transverse dimensions $2 \mathrm{~m} \times 2 \mathrm{~m}$ is distributed throughout the decay volume to capture the decay products from the $A^{\prime}$ decays. It is surrounded by electromagnetic calorimetry designed to efficiently capture the electrons and positrons emergent from the tracking volume (see Figure 9). With these parameters, the yield of detected $A^{\prime} \mathrm{s}$ is marginal - about $5-10$ per $10^{6}$ seconds.

This scenario has not been optimized, and other versions less awkward can be contemplated. However, the region of the exclusion plot that is covered by any such experiment will be modest. Therefore a real design is likely to be opportunistic. If the experiment can be run parasitically for a long period of time, the benefit-to-cost ratio may rise sufficiently high to make such an effort attractive.

If one contemplates utilizing a higher energy dump of 1 MW power, then the number of electrons dumped decreases and, keeping $m_{A^{\prime}}$ and $\epsilon$ fixed, the yield of $A^{\prime}$ s decreases as well. The region of sensitivity at $A^{\prime}$ masses of about $200 \mathrm{MeV}$ should, to a fair approximation, merge with the reach of E137, as estimated in Figure 9 . 

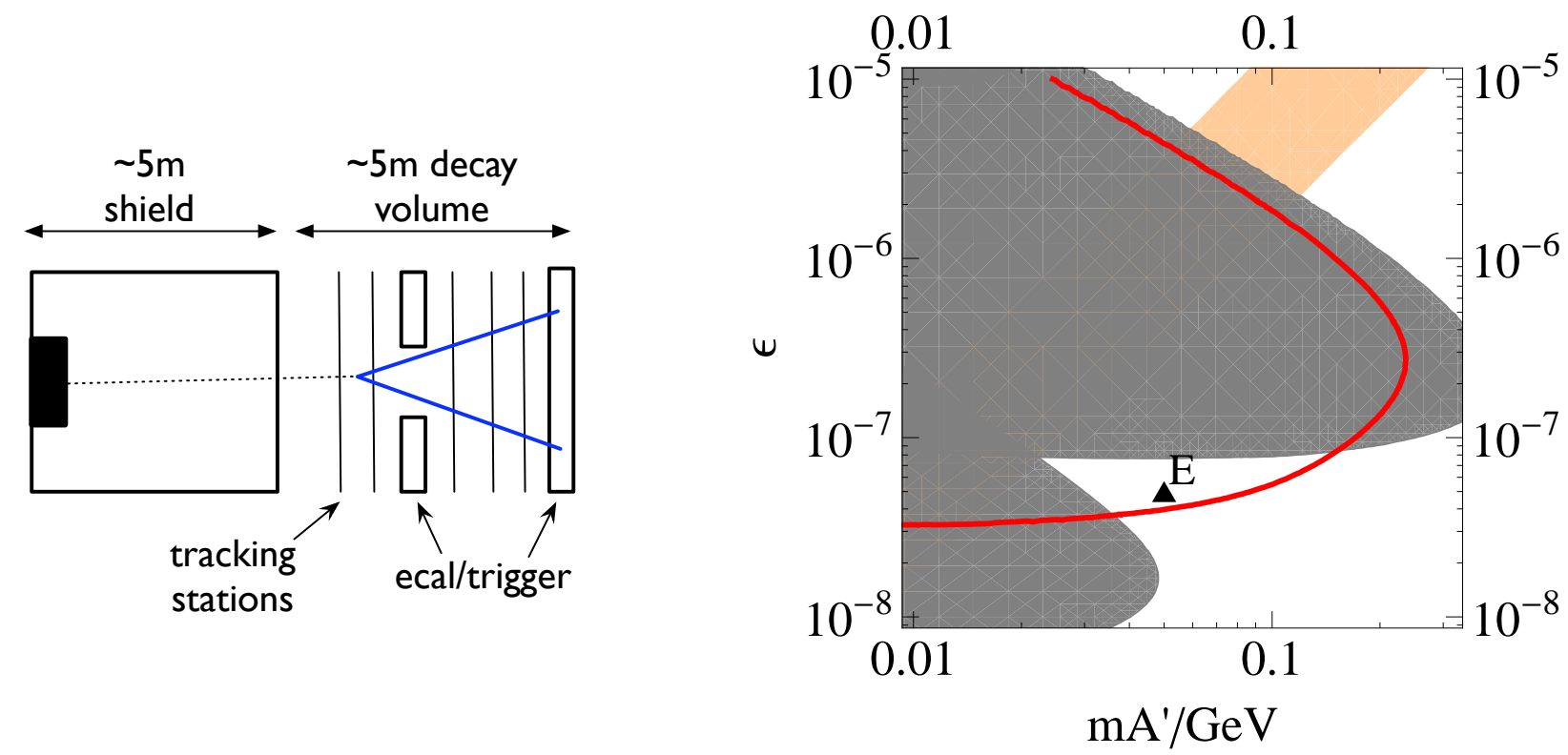

FIG. 9: Left: Schematic diagram of a beam dump design for benchmark point $\mathrm{E}\left(\epsilon \sim 5 \times 10^{-8}, m_{A^{\prime}} \sim 50 \mathrm{MeV}\right)$. A $200 \mathrm{MeV}$ electron beam with a large current of about $5 \mathrm{~mA}$ (delivering 1 megawatt in power) is incident upon a thick tungsten target that together with shielding is about $5 \mathrm{~m}$ in length. Behind the shielding is a decay region $5 \mathrm{~m}$ long, consisting of a tracking system $(2 \mathrm{~m} \times 2 \mathrm{~m}$ transverse to the beam line) and surrounded by electromagnetic calorimeters - see text for further details. Right: Solid Red Contour: 10 events with $A^{\prime}$ energies above $100 \mathrm{MeV}$ after the experiment has run for $10^{6} \mathrm{~s}(5000 \mathrm{C}$ total charge dumped). Gray contours and Orange Stripe: exclusions from past experiments (E137 and SN1987A) and the region that explains DAMA/LIBRA in a simple model — see Figure 1 for more details.

\section{DISCUSSION AND CONCLUSIONS}

In this paper, we have described five scenarios for fixed-target experiments that probe kinetically mixed $U(1)$ 's with $\mathrm{MeV}-\mathrm{GeV}$ masses. Kinetic mixing of size $\epsilon \sim 10^{-2}-10^{-8}$ between a light gauge boson and the photon can be generated by loops of particles at any mass scale, with the magnitude determined by the structure of high-scale physics. An MeV-GeV mass for the $A^{\prime}$ can in turn be generated from the weak scale, especially in a supersymmetric context. An $A^{\prime}$ in this mass range is also of interest as a possible explanation of several current dark matter anomalies.

The parameter space of $A^{\prime}$ mass $m_{A^{\prime}}$ and mixing $\epsilon$ has been constrained from two corners by existing data. Beam dump experiments and supernovas exclude the low-mass, small- $\epsilon$ region. Larger $\epsilon \sim 10^{-3}-10^{-2}$ are constrained for a broad range of masses by lepton anomalous magnetic moments and B-factory searches.

The five approaches we have described cover the remaining parameter space using fixed-target experiments of various geometries and $200 \mathrm{MeV}-6 \mathrm{GeV}$ beams. A natural extension of past beam dumps, with modest intensity and $10 \mathrm{~cm}-1 \mathrm{~m}$ length, can fill in the crevice of parameter space between past beam dumps. Beam dumps are not well suited to searching for $A^{\prime}$ with less displaced decays. For these parameter ranges, thin-target experiments are required.

Any thin-target experiment must contend with the backgrounds from electromagnetic electron scattering and trident production, which can be tackled with a combination of kinematics and displaced vertex selection. Depending on $m_{A^{\prime}}$, more forward or wide-angle geometries are called for, and small-scale silicon microstrip tracking can be utilized to isolate displaced decays. We have considered three such scenarios: a forward two-arm spectrometer, a collinear detector in a diffuse, low-intensity beam, and a wide-angle spectrometer. Together, they are sensitive in the range $\epsilon \sim 10^{-5}-10^{-3}$ for $A^{\prime}$ masses from $10 \mathrm{MeV}$ to $1 \mathrm{GeV}$.

The wide-angle scenario is of particular interest, because existing spectrometers can cover a large fraction of its reach. The Hall A spectrometers and the CLAS detector 51] at JLab seem well suited for initial searches, and other labs may have comparable capabilities.

Searches at low $\epsilon$, below the reach of the dump experiment E137, are limited by practical rate limitations. Power above a megawatt (MW) is difficult to sustain, making $\epsilon \sim 10^{-8}-10^{-7}$ inaccessible with beams of any energy in under a year of running. Our fifth scenario saturates this limit, with a $200 \mathrm{MeV}$ MW dump, which can possibly be accommodated at the JLab Free-Electron Laser accelerator.

When combined with existing limits, these five scenarios can either confirm the existence of new $U(1)$ gauge forces at low masses or close the door on their most likely parameter range.

For masses below the electron threshold, very different 
experimental techniques are called for. These have been developed in 52, 53].

We have restricted our discussion to the simplest scenario: a single $U(1)$ gauge boson that decays directly to electrons. In a larger "dark sector", somewhere between this minimal scenario and the full complexity of Standard Model physics, decays within the dark sector dominate 32. These dark-sector cascades can return some or all of the $A^{\prime}$ energy to Standard Model-charged particles, with lifetimes controlled by $\epsilon^{-2}$ for vector bosons and much longer lifetimes $\propto \epsilon^{-4}$ for scalars. The limits and reaches discussed here apply directly to any spin-1 bosons in the dark sector that decay directly to a lepton pair. These experiments are also sensitive to dark sector cascades involving spin-0 states, with appropriately deformed exclusion regions not discussed here. Besides frameworks with kinetically mixed $U(1)$, these experiments are sensitive to direct production of light (pseudo) scalars (e.g. [54, 55, 56, 57]). It is likely that related designs more optimally cover these scenarios.

We have focused here on experimental approaches tailored to $A^{\prime}$ searches in electron beams, but analyses in this spirit may be possible with existing data, for example by using beam-halo impacts in collider experiments or neutrino production beams and detectors such as those at Fermilab and KEK. We also have not explored the potential of muon-beam experiments, which may be ideal for searches for $A^{\prime}$ s above the muon mass, which are produced with rates comparable to those for an electron beam, but with much lower electromagnetic backgrounds.

\section{Acknowledgments}

We thank Andrei Afanasev, Dan Dale, Alex Dzierba, and Richard Jones for information regarding opportunities at JLab for implementing this program. We are grateful to John Cumalat, Mathew Graham, John Jaros, Aaron Roodman and Jay Wacker for feedback and discussion regarding our experimental scenarios, and to Savas Dimopoulos, Michael Peskin, and Matt Reece for many fruitful discussions. We especially thank Takashi Maruyama for providing us with background estimates for the forward two-arm spectrometer design. RE and PS are supported by the US DOE under contract number DE-AC02-76SF00515.

\section{APPENDIX A: $A^{\prime}$ PRODUCTION FORMULAS}

In this appendix, we first present the cross-section for the production of the massive $U(1)^{\prime}$ "dark photon", $A^{\prime}$, by initial- or final-state radiation off a single electron hitting a fixed target of atomic number $Z$. This process is analogous to photon bremsstrahlung, except that the coupling of the $A^{\prime}$ to electrons is $\epsilon \cdot e$ and the $A^{\prime}$ mass, $m_{A^{\prime}}$, is much larger than the electron mass, $m_{e}$, which significantly alters both the kinematics and the rate of the process. The qualitative behavior has already been summarized in Section II]

We want to calculate the $A^{\prime}$-production cross-section

$$
\frac{d \sigma\left(e(p)+Z\left(P_{i}\right) \rightarrow e\left(p^{\prime}\right)+A^{\prime}(k)+Z\left(P_{f}\right)\right)}{d E_{A^{\prime}} d \cos \theta_{A^{\prime}}}
$$

in the Weizsäcker-Williams approximation following [38, 39, 40, where $k=\left(E_{A^{\prime}}, \vec{k}\right)$ is the momentum of the outgoing $A^{\prime}, \theta_{A^{\prime}}$ is the angle of its momentum relative to the incoming electron momentum $\vec{p}$ in the lab frame, $p=\left(E_{0}, \vec{p}\right)$ and $P_{i}=\left(M_{i}, \overrightarrow{0}\right)$ are the initial momenta of the electron and the target of mass $M_{i}$ and atomic number $Z$, and $p^{\prime}=\left(E^{\prime}, \vec{p}^{\prime}\right)$ and $P_{f}$ are the outgoing four-momenta of the electron and target, which are integrated over.

In the frame of the incoming electron, the rapidly moving atom sources a cloud of effective photons, off which the electron scatters to radiate an $A^{\prime}$. Though these photons are spacelike, their virtuality is small compared to other invariants in the problem (for example $m_{A^{\prime}}$ ), so that the interaction of the electron with the target is dominated by transverse polarizations. Therefore, it is related to the cross-section for real-photon scattering, $e(p) \gamma(q) \rightarrow e\left(p^{\prime}\right) A^{\prime}(k)$ with $q=P_{i}-P_{f}$ by 40]

$$
\begin{gathered}
\frac{d \sigma\left(p+P_{i} \rightarrow p^{\prime}+k+P_{f}\right)}{d E_{A^{\prime}} d}=\left(\frac{\alpha \chi}{\pi}\right)\left(\frac{E_{0} x \beta_{A^{\prime}}}{(1-x)}\right) \\
\times\left.\frac{d \sigma\left(p+q \rightarrow p^{\prime}+k\right)}{d(p \cdot k)}\right|_{t=t_{\text {min }}},
\end{gathered}
$$

where

$$
\begin{aligned}
x & \equiv E_{A^{\prime}} / E_{0}, \\
t & \equiv-q^{2} .
\end{aligned}
$$

We specify the kinematics at $t=t_{\text {min }}$ and the effective photon flux $\frac{\alpha \chi}{\pi}$ below. Note that $t$ is not one of the Mandelstam variables for the $2 \rightarrow 2$ process, which will be denoted by $t_{2}$ - see below.

For a given $A^{\prime}$ four-momentum $k$, the virtuality $t$ has its minimum value $t_{\text {min }}$ when $\vec{q}$ is collinear with the threevector $\vec{k}-\vec{p}$. Solving the mass-shell conditions $p^{2}=$ $(q+p-k)^{2}=m_{e}^{2}$ and $P_{f}^{2}=\left(P_{i}-q\right)^{2}=M_{i}^{2}$ with the collinear geometry, and keeping only leading effects in

$$
\frac{m_{A^{\prime}}^{2}}{E_{k}^{2}}, \quad \frac{m_{e}^{2}}{E^{\prime 2}}, \quad \theta_{A^{\prime}}, \quad \frac{|\vec{q}|}{E^{\prime}},
$$

(with $|\vec{q}|$ defined below), we find

$$
\begin{aligned}
& q^{0}=|\vec{q}|^{2} / 2 M_{i} \approx 0, \quad|\vec{q}|=\frac{U}{2 E_{0}(1-x)}, \\
& t_{\text {min }}=-q_{\text {min }}^{2} \approx\left(\frac{U}{2 E_{0}(1-x)}\right)^{2},
\end{aligned}
$$

where

$$
U \equiv U\left(x, \theta_{A^{\prime}}\right)=E_{0}^{2} \theta_{A^{\prime}}^{2} x+m_{A^{\prime}}^{2} \frac{1-x}{x}+m_{e}^{2} x
$$


At this kinematics,

$$
\begin{aligned}
-\tilde{u} & \equiv m_{e}^{2}-u_{2}=2 p \cdot k-m_{A^{\prime}}^{2}=U, \\
\tilde{s} & \equiv s_{2}-m_{e}^{2}=2 p^{\prime} \cdot k+m_{A^{\prime}}^{2}=\frac{U}{1-x}, \\
t_{2} & =\left(p-p^{\prime}\right)^{2}=-\frac{U x}{1-x}+m_{A^{\prime}}^{2}
\end{aligned}
$$

where $s_{2}, t_{2}$, and $u_{2}$ are the Mandelstam variables for the $2 \rightarrow 2$ process. The cross-section for the $2 \rightarrow 2$ process is therefore

$$
\begin{aligned}
\frac{d \sigma}{d(p \cdot k)}=2 \frac{d \sigma}{d t_{2}} \approx \frac{1}{8 \pi\left(s_{2}-m_{e}^{2}\right)^{2}}|\mathcal{M}|^{2} & =\frac{4 \pi \alpha^{2} \epsilon^{2}}{\tilde{s}^{2}}\left(\frac{\tilde{s}}{-\tilde{u}}+\frac{-\tilde{u}}{\tilde{s}}+\frac{2 m_{A^{\prime}}^{2} t_{2}}{-\tilde{u} \tilde{s}}\right) \\
& =\left(4 \pi \alpha^{2} \epsilon^{2}\right) \frac{(1-x)}{U^{2}}\left[1+(1-x)^{2}+\frac{2(1-x)^{2} m_{A^{\prime}}^{2}}{U^{2}}\left(m_{A^{\prime}}^{2}-\frac{U x}{1-x}\right)\right]
\end{aligned}
$$

where we have dropped the $t$-dependence of $\frac{d \sigma}{d t_{2}}$ and terms of order $m_{e}^{2}$ in $|\mathcal{M}|^{2}$. Therefore, the Weizsäcker-Williams approximation to the cross-section A1 is given by

$$
\frac{1}{E_{0}^{2} x} \frac{d \sigma_{3 \rightarrow 2}}{d x d \cos \theta_{A^{\prime}}}=\left(8 \alpha^{3} \epsilon^{2} \chi \beta_{A^{\prime}}\right)\left[\frac{1-x+\frac{x^{2}}{2}}{U^{2}}+\frac{(1-x)^{2} m_{A^{\prime}}^{2}}{U^{4}}\left(m_{A^{\prime}}^{2}-\frac{U x}{1-x}\right)\right]
$$

where $\beta_{A^{\prime}} \equiv \sqrt{1-m_{A^{\prime}}^{2} / E_{0}^{2}}$. The $x$-differential crosssection is obtained by integrating $\mathrm{A} 12$ with respect to $\theta_{A^{\prime}}$ (we will see below that $\chi$ actually depends on $\theta_{A^{\prime}}$, but this can be neglected to excellent approximation). The first term in square brackets integrates to

$$
\frac{1-x+\frac{x^{2}}{2}}{U\left(x, \theta_{A^{\prime}}=0\right)} \text {. }
$$

In the limit $m_{A^{\prime}} \rightarrow 0$, this becomes the standard photon bremsstrahlung result with a $\frac{1}{x}$ singularity, while the second term in the square brackets vanishes. However, finite $m_{A^{\prime}}$ regulates this singularity, and in the case of interest, namely $m_{A^{\prime}} \gg m_{e}$, we have $U(x, 0) \approx m_{A^{\prime}}^{2} \frac{1-x}{x}$. The second term integrates to $-\frac{x^{2}}{6 U(x, 0)}+\mathcal{O}\left(m_{e}^{2} U^{2}\right)$, so that

$$
\begin{aligned}
\frac{d \sigma_{3 \rightarrow 2}}{d x}= & \left(8 \alpha^{3} \epsilon^{2} \chi \beta_{A^{\prime}}\right)\left(m_{A^{\prime}}^{2} \frac{1-x}{x}+m_{e}^{2} x\right)^{-1} \\
& \times\left(1-x+\frac{x^{2}}{3}\right) .
\end{aligned}
$$

This has an approximate soft electron singularity, regulated by the electron mass at $(1-x)_{c 1}=\frac{m_{e}^{2}}{m_{A^{\prime}}^{2}}$. Though not explicit in this formula, our approximations also break down if the electron energy $(1-x) E_{0} \lesssim|\vec{q}|$; this also regulates the cross-section, cutting off $\log (1-x)$ at $(1-x)_{c 2}=\frac{m_{A^{\prime}}^{2}}{E_{0}^{2}}$. Since one cutoff or the other is always larger than their geometric mean $m_{e} / E_{0}$, the $A^{\prime}$ is always produced from a relativistic electron. The $x$-integrated cross-section is therefore

$$
\begin{aligned}
& \sigma \approx \frac{8}{3} \frac{\alpha^{3} \epsilon^{2} \beta_{A^{\prime}}}{m_{A^{\prime}}^{2}} \chi \log \left(\frac{1}{(1-x)_{c}}\right), \\
& (1-x)_{c}=\max \left(\frac{m_{e}^{2}}{m_{A^{\prime}}^{2}}, \frac{m_{A^{\prime}}^{2}}{E_{0}^{2}}\right) .
\end{aligned}
$$

As we have noted in Section II the characteristic angle of $A^{\prime}$ emission is set by $U\left(x, \theta_{A^{\prime}}\right)-U(x, 0) \sim U(x, 0)$, so $\theta_{A^{\prime}} \sim \frac{m_{A^{\prime}} \sqrt{\overline{1-x}}}{E_{0}}$, where the median value of $1-x$ is $\overline{1-x} \sim \max \left(\frac{m_{e}}{m_{A^{\prime}}}, \frac{m_{A^{\prime}}}{E_{0}}\right)$. This is parametrically smaller than the angle of the $A^{\prime}$ decay products with respect to the incoming electron, namely $\sim m_{A^{\prime}} / E_{0}$.

We turn next to the definition of $\chi$, which is an effective flux of photons integrated from $t=t_{\min }$ to $t_{\max }$, the total center-of-mass energy of the collision. We refer the reader to [38, 39] for more details.

For a general electric form factor $G_{2}(t)$,

$$
\chi \equiv \int_{t_{\min }}^{t_{\max }} d t \frac{t-t_{\min }}{t^{2}} G_{2}(t) .
$$

(We note that the other form factor, $G_{1}(t)$, contributes only a negligible amount in all cases of interest.) Although the virtual photon propagator squared, $1 / t^{2}$, is 
dominated at $t=t_{\text {min }}$, the final-state phase space is proportional to $d t\left(t-t_{\text {min }}\right)$, so that virtual photons at all scales contribute to $A^{\prime}$ production. As discussed in 38, 39, the physical upper bound may be set not by the center-of-mass energy, but by $t_{\max } \sim m_{A^{\prime}}^{2}$, at which the full $2 \rightarrow 3$ matrix element begins to shut off.

For most energies in question, $G_{2}(t)$ is dominated by an elastic component

$$
G_{2, e l}(t)=\left(\frac{a^{2} t}{1+a^{2} t}\right)^{2}\left(\frac{1}{1+t / d}\right)^{2} Z^{2}
$$

where the first term parametrizes electron screening (the elastic atomic form factor) with $a=111 Z^{-1 / 3} / m_{e}$, and the second finite nuclear size (the elastic nuclear form factor) with $d=0.164 \mathrm{GeV}^{2} A^{-2 / 3}$. We have multiplied together the simple parametrizations used for each in [38. The logarithm from integrating (A17) is large for $t_{\min }<d$, which is true for most of the range of interest. However, for heavy $A^{\prime}$, the elastic contribution is suppressed and is comparable to an inelastic term,

$$
G_{2, i n}(t)=\left(\frac{a^{\prime 2} t}{1+a^{\prime 2} t}\right)^{2}\left(\frac{1+\frac{t}{4 m_{p}^{2}}\left(\mu_{p}^{2}-1\right)}{\left(1+\frac{t}{0.71 \mathrm{GeV}^{2}}\right)^{4}}\right)^{2} Z,
$$

where the first term parametrizes the inelastic atomic form factor and the second the inelastic nuclear form factor, and where $a^{\prime}=773 Z^{-2 / 3} / m_{e}, m_{p}$ is the proton mass, and $\mu_{p}=2.79$ [38]. This expression is valid when $t / 4 m_{p}^{2}$ is small, which is the case for $m_{A^{\prime}}$ in the range of interest in this paper. One can show that the contribution from the other inelastic nuclear form factor $G_{1}(t)$ is negligible.

At high masses, these simple parameterizations of the form factors are uncertain at the order-of-magnitude level. Using $G_{2, e l}+G_{2, \text { in }}$ in (A17), and setting $t_{\text {min }}=$ $\left(m_{A^{\prime}}^{2} / 2 E_{0}\right)^{2}, t_{\max }=m_{A^{\prime}}^{2}$, we obtain $\chi / Z^{2}$ shown in Figure 10.

\section{APPENDIX B: PRODUCTION FROM THIN AND THICK TARGETS OF $A^{\prime}$ S WITH FINITE LIFETIME}

Consider an electron beam with energy $E_{0}$ incident on a target. The total number of $A^{\prime}$ that get produced in the target with energy $E_{A^{\prime}} \equiv x E_{0}$ and decay at a distance $z$ behind the front edge of the target is given by

$$
\begin{aligned}
\frac{d N}{d x d z}= & N_{e} \frac{N_{0} X_{0}}{A} \int_{E_{A^{\prime}}}^{E_{0}} d E_{1} \int_{0}^{T} d t I\left(E_{1} ; E_{0}, t\right) \\
& \times\left(\frac{E_{0}}{E_{1}} \frac{d \sigma}{d x^{\prime}}\right)_{x^{\prime}=\frac{E_{A^{\prime}}}{E_{1}}} \frac{d P\left(z-\frac{X_{0}}{\rho} t\right)}{d z},
\end{aligned}
$$

where $E_{0}$ is the incident energy, $N_{e}$ the number of incident electrons, $N_{0}=6.02 \times 10^{23} \mathrm{~mole}^{-1}, \rho$ and $X_{0}$ are the

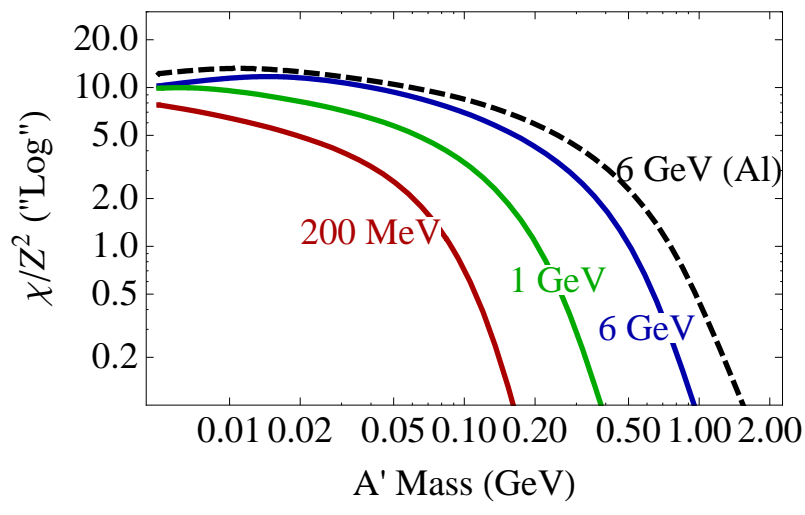

FIG. 10: The factor $\mathcal{L} o g=\chi / Z^{2}$ appearing in equations and $(8)$ in Section II Solid lines from left to right correspond to beams of energy $200 \mathrm{MeV}, 1 \mathrm{GeV}$, and $6 \mathrm{GeV}$, respectively, incident on a Tungsten target. The dashed line corresponds to a $6 \mathrm{GeV}$ beam incident on an Aluminum target.

density (in $\mathrm{g} / \mathrm{cm}^{3}$ ) and unit radiation length (in $\mathrm{g} / \mathrm{cm}^{2}$ ) of the target material, respectively, and

$$
\frac{d P(\ell)}{d \ell}=\frac{1}{\ell_{0}} e^{-\ell / \ell_{0}}
$$

is the differential decay probability where $\ell_{0} \equiv \gamma c \tau=$ $E_{1} c \tau / m_{A^{\prime}}$ is the $A^{\prime}$ decay length given in $(12)$. Also,

$$
I\left(E_{1} ; E_{0}, t\right) \approx \begin{cases}\frac{1}{E_{0}} y^{b t-1} b t & T \gtrsim 1 \\ \delta\left(E_{1}-E_{0}\right) & T \ll 1\end{cases}
$$

is the energy distribution of electrons at position $t$ in the target, where $y \equiv \frac{E_{0}-E_{1}}{E_{0}}$ and $b=4 / 3$.

We can perform the $t$-integration explicitly in the limit of a very thin or thick target $(T \ll 1$ or $T \gg 1)$. For a thin target, we find

$$
\frac{d N_{\text {thin }}}{d x d z}=N_{e} \frac{N_{0} \rho \ell_{0}}{A} \frac{d \sigma}{d x}\left(e^{\frac{T X_{0}}{\rho \ell_{0}}}-1\right) \frac{d P(z)}{d z} .
$$

For a thick target, we neglect the $t$-dependence in the $A^{\prime}$ decay probability in (B1), since most production occurs within the first radiation length and thus well before the end of the dump. Here we find

$$
\begin{aligned}
\frac{d N_{\text {thick }}}{d x d z} \approx \quad & N_{e} \frac{N_{0} X_{0}}{A} \int_{E_{A^{\prime}}}^{E_{0}} d E_{1} \tilde{I}\left(E_{1} ; E_{0}, T\right) \\
& \times\left(\frac{E_{0}}{E_{1}} \frac{d \sigma}{d x^{\prime}}\right)_{x^{\prime}=\frac{E_{A^{\prime}}}{E_{1}}} \frac{d P(z)}{d z},
\end{aligned}
$$

where

$$
\begin{aligned}
& \tilde{I}\left(E_{1} ; E_{0}, T\right)=\int_{0}^{T} d t I\left(E_{1} ; E_{0}, t\right) \\
& \quad \approx \frac{1+y^{b T}(b T \ln y-1)}{E_{0} b y(\ln y)^{2}} \rightarrow \frac{1}{E_{0} b y(\ln y)^{2}}
\end{aligned}
$$


as $T \rightarrow \infty$. For finite $T$, the limiting form is a good approximation for small and moderate $y$, i.e. for electrons that carry a large fraction of the initial beam energy (for $y<0.5$ and $T>7$ it is correct to within $1 \%$ ). to

We note that by (8) and (B1), $\frac{1}{N_{e}} \frac{d N}{d x d z}$ is proportional

$$
\frac{1}{\ell_{0}} \frac{8 \alpha^{3} \epsilon^{2} Z^{2} \chi}{m_{A^{\prime}}^{2}} \frac{N_{0} X_{0}}{A} .
$$

However, $\frac{1}{X_{0}}=\frac{4 \alpha^{3} N_{0}}{m_{e}^{2} A}\left[Z^{2}\left(L_{\text {rad }}-f(Z)\right)+Z L_{\text {rad }}^{\prime}\right]$, where $L_{\text {rad }}, L_{\text {rad }}^{\prime}$, and $f$ are logs set by the atomic form factors of the target atoms [41, and we obtain

$$
\frac{d N}{d x d z} \sim \frac{\min (T, 1)}{\ell_{0}} \frac{m_{e}^{2}}{m_{A^{\prime}}^{2}} \epsilon^{2},
$$

with only logarithmic dependence on the target nucleus $Z$. This expression has a simple physical interpretation: an electron is slowed in a radiation length by radiating a small number of relatively hard but collinear photons. The probability of instead radiating an $A^{\prime}$ is suppressed by the squared ratio of the couplings, $\epsilon^{2}$, and the squared ratio of the masses $\frac{m_{e}^{2}}{m_{A^{\prime}}^{2}}$, because it requires a higher invariant-mass intermediate state.

For decays at a given $z$, we applied an acceptance based on the detector geometry and energy cuts before integrating to obtain the expected total rate.

\section{APPENDIX C: KINEMATICS OF SIGNAL AND CONTROLLING BACKGROUNDS}

The dominant QED backgrounds for $A^{\prime}$ production are the trident reactions shown in Figure 3. The $\gamma^{*}$ process contributes an irreducible background to $A^{\prime} \rightarrow l^{+} l^{-}$. The Bethe-Heitler process has a much larger rate, but can be controlled by exploiting its very different kinematics compared to the signal. Our aim in this appendix is to quantitatively describe the singularity structure of the Bethe-Heitler process and derive an effective set of cuts on lab-frame observables. Of course a more accurate basis for a final design would rely on monte carlo for these processes, but the simple calculation clarifies the origin of the large Bethe-Heitler cross-section and how to regulate it by cutting away from the dangerous "forward" and "asymmetric" regions of phase space in the lab frame.

As an important reference, we will start by recalling the kinematic properties of $A^{\prime}$ production and decay using the results of Appendix A. We again consider a monochromatic incident electron beam of energy $E_{0}$. Let $\theta_{c m}$ be the emission angle of the forward decay product relative to the $A^{\prime}$ direction in the $A^{\prime}$ rest frame. Let $\theta_{A^{\prime}}$ be the emission angle of the $A^{\prime}$ relative to the beam direction in the lab frame. As we have shown, the characteristic $A^{\prime}$ emission angle is small and is set by $\theta_{A^{\prime}} \sim \frac{m_{A^{\prime}} \sqrt{\overline{1-x}}}{E_{0}}$, where $x \equiv E_{A^{\prime}} / E_{0}$. In the limit of small $\frac{m_{A^{\prime}}}{x E_{0}}$, the lab frame opening angles $\theta_{ \pm}$and energies $E_{ \pm}$of the $A^{\prime}$ decay products are,

$$
\begin{aligned}
E_{ \pm} & =\frac{x E_{0}}{2}\left(1 \pm \cos \left(\theta_{c m}\right)\right) \\
\tan \left(\theta_{ \pm}\right) & = \pm \frac{1}{\gamma} \sqrt{\frac{1 \mp \cos \left(\theta_{c m}\right)}{1 \pm \cos \left(\theta_{c m}\right)}}+\tan \left(\theta_{A^{\prime}}\right)
\end{aligned}
$$

where $\gamma=\frac{x E_{0}}{m_{A^{\prime}}}$.

The characteristic transverse momentum of the $A^{\prime}$ is $p_{A^{\prime}, \perp} \approx E_{A^{\prime}} \theta_{A^{\prime}} \sim \sqrt{1-x} m_{A^{\prime}}$, while the typical recoil of the target is $\left|q_{\text {min }}\right| \approx\left(\frac{m_{A^{\prime}}}{2 x E_{0}}\right) m_{A^{\prime}}$. The median value of $1-x$ is $\overline{1-x} \approx \frac{m_{A^{\prime}}}{E_{0}}$ for $m_{A^{\prime}} \gtrsim 50 \mathrm{MeV}$, implying that $\left|q_{\text {min }}\right|$ is parametrically smaller than $p_{A^{\prime}, \perp}$ by $\sim$ $\sqrt{\frac{m_{A^{\prime}}}{E_{0}}}$. Evidently, the recoiling electron largely balances the recoil of the $A^{\prime}$. The energy $E_{R}$ and angle $\theta_{R}$ of the recoiling final state beam electron in the laboratory frame is,

$$
\begin{aligned}
E_{R} & =(1-x) E_{0} \approx m_{A^{\prime}} \\
\tan \left(\theta_{R}\right) & \approx \sqrt{\frac{m_{A^{\prime}}}{E_{0}}}\left(1+\frac{m_{A^{\prime}}}{2 E_{0}}+\ldots\right) .
\end{aligned}
$$

Note the relatively wide angle of the recoiling electron relative to the $A^{\prime}$ decay products. Equations (C1)-(C4) summarize the important kinematic characteristics of $A^{\prime}$ production.

As with $A^{\prime}$ production, trident reactions can also be analyzed using the Weizsäcker-Williams approximation, where we group an outgoing $\ell^{+} \ell^{-}$pair with fixed invariant mass $m^{2}$ to act as the $A^{\prime}$ candidate. In the case of Bethe-Heitler production, we can further approximate the beam electron as splitting at small angle into the recoil electron and a nearly on-shell photon, which scatters with the Coulomb photon into the $\ell^{+} \ell^{-}$pair (see Figure 11. There are two sources of large logs in the Bethe-Heitler cross-section: the soft and collinear logs in the photon radiation by the electron and a forwardscattering $\log$ in the $\gamma \gamma \rightarrow \ell^{+} \ell^{-}$subprocess. Both are regulated by $m_{e}$, and can be further suppressed by kinematic requirements. We postpone the derivation - a matter of standard results and kinematic bookkeeping - to the following section, and focus here on its implications.

In this approximation, upon integrating over an invariant mass window of size $\delta m$ about $m_{A^{\prime}}$, we find

$$
\begin{aligned}
\frac{d \sigma}{d x d \cos \theta_{A^{\prime}} d \hat{c}}=\frac{2 \alpha^{4} \chi}{\pi} & \frac{\delta m}{m_{A^{\prime}}} \frac{1}{m_{A^{\prime}}^{2}} \frac{1+(1-x)^{2}}{\theta_{A^{\prime}}^{2} x} \\
& \times\left(\frac{1+\hat{c}}{1-\hat{c}}+\frac{1-\hat{c}}{1+\hat{c}}\right),
\end{aligned}
$$

where $x$ and $\theta_{A^{\prime}}$ are defined as before, and $\hat{c}=\cos \theta_{c m}$, where $\theta_{c m}$ is measured relative to the axis of the incoming photons in the $\gamma \gamma \rightarrow \ell^{+} \ell^{-}$process, which is near enough to the beam axis for our purposes. This displays the 
expected singularities at small $x$ (soft), $|\hat{c}| \rightarrow 1$ (forward scattering), and small $\theta_{A^{\prime}}$ (collinear). This is quite different behavior from the signal and radiative backgrounds, which are peaked at large values of $x$ and slowly varying in $\hat{c}$.

Requiring $x$ above $1-\delta$, with $\delta$ near or below its median value $\bar{\delta}=\max \left(m_{A^{\prime}} / E_{0}, m_{e} / m_{A^{\prime}}\right)$ keeps a large fraction of the signal and suppresses the Bethe-Heitler background by a factor of $\delta$. Likewise, the signal is relatively flat in $\hat{c}=\cos \theta_{c m}$. According to (C1) and (C2), we can ensure modest $\hat{c}$ by constraining the ratio of the labframe energies or of the opening angles of the two decay products to be near unity.

There remains the collinear singularity at small $\theta_{A^{\prime}}$. The signal is also peaked forward, but with a singularity regulated by $m_{A^{\prime}}$ rather than $m_{e}$, so that it is produced at much wider characteristic $A^{\prime}$ angles than Bethe-Heitler processes. Since the absolute angles $\sim\left(m_{A^{\prime}} / E_{0}\right)^{3 / 2}$ are still small and must be obtained by summing two momenta, it is impractical to place a lower bound on the $A^{\prime}$ emission angle. It is probably much easier to cut on the angle $\theta_{R}$ of the recoiling electron. Since the recoiling electron has much lower energy at large $x$ and approximately balances the transverse momentum of the $A^{\prime}$, it is emitted at a much larger angle. For the median $x$, the electron energy $\sim m_{A^{\prime}}$ and its angle $\sim\left(m_{A^{\prime}} / E_{0}\right)^{1 / 2}$. Requiring the recoiling electron momentum and angle near these values significantly reduces the Bethe-Heitler rate. In a similar spirit, it may or may not also be easier to implement a tight $x$ cut using the energy of the recoil electron rather than the total energy of the decay products making up the $A^{\prime}$ candidate. After these cuts, we find

$$
\begin{aligned}
\sigma_{c u t} & \left(x_{\min }, \theta_{R, \min },|\hat{c}|_{\max }\right) \approx \frac{16 \alpha^{4} \chi}{\pi} \frac{\delta m}{m_{A^{\prime}}} \frac{1}{m_{A^{\prime}}^{2}} \\
& \times \log \left(\left(1-x_{\min }\right)^{-1} \theta_{R, \min }^{-1}\right)\left(1-x_{\min }\right) \\
& \times\left(\tanh ^{-1}|\hat{c}|_{\max }-|\hat{c}|_{\max } / 2\right) .
\end{aligned}
$$

When $\theta_{\text {cut }}$ is small relative to the typical angular spread $\frac{m_{A^{\prime}}}{E_{0}}(1-x)$ of the signal and radiative backgrounds, the factor on the first line is related to the lepton-pair crosssection $\sigma_{r a d}$ with the same cuts from the radiative diagrams alone by

$$
9 \sigma_{\text {rad }}\left(\log \frac{1-x_{\text {min }}}{1-x_{\max }}\right)^{-1} \epsilon_{\text {rad }}^{-1}\left(\theta_{R, \min },|\hat{c}|_{\text {max }}\right),
$$

where $1-x_{\max }=\max \left(m_{A}^{2} / E_{0}^{2}, m_{e}^{2} / m_{A}^{2}\right)$ is the value of $x$ where the $\log$ divergence in $\sigma_{\text {rad }}$ is regulated, and $\epsilon_{\text {rad }}$ the efficiency for the radiative process (or signal) to pass $\theta_{R}$ and $|\hat{c}|$ selections.

Let us consider a representative case, $E_{0}=5 \mathrm{GeV}$, $m_{A^{\prime}}=0.5 \mathrm{GeV}$. Requiring $x>0.9, \theta_{R}>1 / 10$, and $E_{+} / E_{-}<3$ (i.e. $\hat{c}<0.5$ ), we retain roughly $20 \%$ of the signal and reduce the contribution of Bethe-Heitler to the signal region to roughly the same size as the radiative contribution. A proper optimization of these cuts

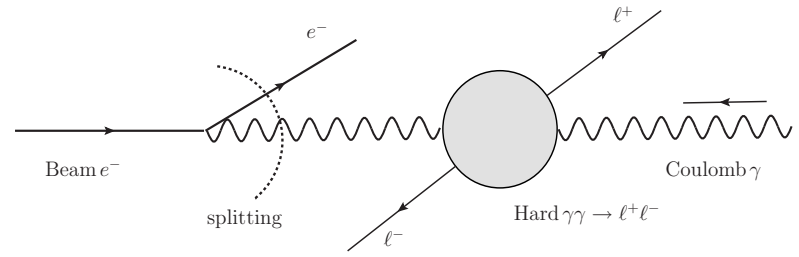

FIG. 11: Bethe-Heitler reactions viewed as hard $\gamma \gamma \rightarrow l^{+} l^{-}$ processes.

is best done with full monte carlo for the background, and of course depends on the characteristics of an individual experiment, but we have confirmed numerically that the kinematic differences between Bethe-Heitler and radiative production are sufficient that it can be made sub-dominant while maintaining high efficiency for the $A^{\prime}$ signal.

In the case of $A^{\prime} \rightarrow e^{+} e^{-}$, the Bethe-Heitler process can also contribute with the electron labeled $\ell^{-}$in Figure 3 identified as the recoiler and $\ell^{+}$and $e^{-}$forming the $A^{\prime}$ candidate. Here the recoil electron kinematics is as in the signal process but there is a forward-scattering singularity when most of the $A^{\prime}$-candidate energy is carried by the $e^{-}$with a softer $e^{+}$. The cuts above remove this singularity as well, and we will not discuss it further.

\section{Bethe-Heitler Pair Production in the Collinear Splitting Approximation}

We now extend the earlier Weizsacker-Williams treatment to compute the Bethe-Heitler pair production crosssection. Using the notation defined in Appendix A, but now with an outgoing lepton/anti-lepton pair with momenta $l^{-} / l^{+}$, the fully differential cross section is,

$$
\begin{gathered}
\frac{d \sigma\left(p_{1}+P_{i} \rightarrow p_{2}+l^{+}+l^{-}+P_{f}\right.}{d E_{A^{\prime}} d \cos \theta_{A^{\prime}} d m^{2} d \hat{t} d \phi_{d}}=\left(\frac{\alpha \chi}{\pi}\right)\left(\frac{E_{0} x \beta_{A^{\prime}}}{(1-x)}\right) \\
\times\left.\frac{d \sigma\left(p_{1}+q \rightarrow p_{2}+l^{+}+l^{-}\right)}{d\left(p_{1} \cdot a\right) d m^{2} d \hat{t} d \phi_{d}}\right|_{t=t_{\text {min }}}
\end{gathered}
$$

where $a=l^{+}+l^{-}$is the total four-momentum of the $A^{\prime}$ candidate, $m^{2}=a^{2}$ their invariant mass, and $\hat{t}=$ $\left(l^{+}-q\right)^{2}=\left(l^{+}-P_{f}+P_{i}\right)^{2} . \phi_{d}$ the angle between $p_{1}$ and $l^{+}$in the rest frame of $a$. As before, the hard subprocesses ( $2 \rightarrow 3$ in this case) are separated from the soft Coulomb photon exchange.

Starting from equation (C8), we will analyze the behavior of Bethe-Heitler reactions (see Figure 3 (b)) relative to $A^{\prime}$ production in a leading logarithm approximation. This suffices to identify the singularities.

To write the right-hand side of (C8) in the approximation of near-collinear splitting, it is useful to introduce the more familiar $z$ and $p_{\perp}$ variables and relate them to the kinematic variables in the center-of-mass frame of the beam electron and Coulomb photon and to the lab-frame. We recall that in the lab frame, the 
Coulomb photon is purely spacelike, with $|\vec{q}|=\sqrt{t_{\min }}=$ $\frac{U\left(x, \theta_{A^{\prime}}\right)}{2 E_{0}(1-x)} \approx m^{2} /\left(2 E_{0} x\right)$ for small $\theta_{A^{\prime}}$ (we have replaced $m_{A^{\prime}}^{2}$ with the invariant mass $m^{2}$ for the off-shell processes, but the kinematic conditions are unchanged). As $|\vec{q}|$ at $t_{\text {min }}$ kinematics depends on $x$ and $\theta_{A^{\prime}}$, the CM frame does too - it is obtained from the $\mathrm{CM}$ frame by boosting with $\beta \approx 1-q / E_{0}$ (we drop much smaller $\theta_{A^{\prime-}}$ dependent corrections), leading to CM-frame momenta for the incoming electron and Coulomb photon,

$$
\begin{aligned}
p_{1}^{c m} & =(p, 0,0, p) \\
q^{c m} & =(\beta p, 0,0,-p)
\end{aligned}
$$

where $p=\sqrt{\frac{q E}{2(1-q / E)}}$. The recoil electron has momentum

$$
\begin{aligned}
p_{2}^{l a b}= & \left((1-x) E_{0}, E_{0} \theta_{A^{\prime}} x, 0\right. \\
& \left.(1-x) E_{0}-\frac{E_{0} \theta_{A^{\prime}}^{2} x^{2}}{2(1-x)}\right), \\
p_{2}^{c m}= & \left(z p, p_{\perp}, 0, \sqrt{z^{2} p^{2}-p_{\perp}^{2}}\right)
\end{aligned}
$$

where

$$
z \equiv 1-x, \quad p_{\perp} \equiv E_{0} \theta_{A^{\prime}} x
$$

up to corrections of higher order in $\theta_{A^{\prime}}$ and $m_{e}$. For small $p_{\perp}$, we can now approximate

$$
\frac{d \sigma}{d z d p_{\perp}^{2} d \hat{t} d \phi_{d}}=\left(\frac{\alpha}{2 \pi}\right) \frac{\left[1+z^{2}\right]}{(1-z) p_{\perp}^{2}} \frac{d \sigma\left(\gamma \gamma \rightarrow l^{+} l^{-}\right)}{d \hat{t} d \phi_{d}}
$$

where

$$
\frac{d \sigma\left(\gamma \gamma \rightarrow l^{+} l^{-}\right)}{d \hat{t} d \phi_{d}}=\frac{\alpha^{2}}{m^{4}}\left(\frac{\hat{t}}{\hat{u}}+\frac{\hat{u}}{\hat{t}}\right)
$$

We emphasize that the recoil electron is always rightmoving, so the splitting approximation is valid where the Bethe-Heitler cross-section is largest, but is never a good approximation for the radiative (or signal) processes, where the $\gamma^{*} \rightarrow e^{-} e^{+*}$ "splitting" always produces an electron going backwards relative to the $\gamma^{*}$.

The kinematic variables of $\mathrm{C} 8$ are related to $z, p_{\perp}, \phi_{d}$ by

$$
\begin{aligned}
m^{2} & =a^{2}=(1+\beta-2 z)(1+\beta) p^{2} \\
p_{1} \cdot a & =(1+\beta-z) p^{2}+z p^{2} \sqrt{1-\frac{p_{\perp}^{2}}{z^{2} p^{2}}} \\
\phi_{d} & =\phi_{d},
\end{aligned}
$$

leading to a Jacobian factor

$$
\frac{d^{2}}{d\left(p_{1} \cdot a\right) d m^{2}} \approx \frac{z}{p^{2}(1+\beta)} \frac{d^{2}}{d p_{\perp}^{2} d z} \approx \frac{2 x(1-x)}{m^{2}} \frac{d^{2}}{d p_{\perp}^{2} d z}
$$

Taking $1+\beta \rightarrow 2, p^{2} \rightarrow \frac{|\vec{q}| E_{0}}{2} \approx \frac{m^{2}}{4 x}$, we find

$$
\left.\frac{d \sigma\left(p_{1}+q \rightarrow p_{2}+l^{+}+l^{-}\right)}{d\left(p_{1} \cdot a\right) d m^{2} d \hat{t} d \phi_{d}}\right|_{t=t_{\min }\left(x, \theta_{A^{\prime}}\right)}=\frac{\alpha}{2 \pi} \frac{1+(1-x)^{2}}{E_{0}^{2} \theta_{A^{\prime}}^{2} x^{3}} \frac{2 x(1-x)}{m^{2}} \frac{\alpha^{2}}{m^{4}}\left(\frac{\hat{t}}{\hat{u}}+\frac{\hat{u}}{\hat{t}}\right),
$$

and hence by (C8),

$$
\frac{d \sigma}{d x d \cos \theta_{A^{\prime}} d m^{2} d \hat{t} d \phi_{d}}=\frac{\alpha^{4} \chi}{\pi^{2}} \frac{1+(1-x)^{2}}{\theta_{A^{\prime}}^{2} x} \frac{1}{m^{6}}\left(\frac{\hat{t}}{\hat{u}}+\frac{\hat{u}}{\hat{t}}\right) .
$$

Changing variables from $\hat{t}$ to $\hat{c}=\cos \theta_{c m}$ of the $2 \rightarrow 2$ process, $\hat{t}=\frac{m^{2}}{2}(1-\hat{c})$, and integrating over $\phi_{d}$ and over $m^{2}$ from $m_{A^{\prime}}^{2}-m_{A^{\prime}} \delta m$ to $m_{A^{\prime}}^{2}+m_{A^{\prime}} \delta m$, we obtain the result of Equation $\mathrm{C} 5$.
[1] B. Holdom, Phys. Lett. B166, 196 (1986).

[2] N. Arkani-Hamed and N. Weiner, JHEP 12, 104 (2008), 0810.0714 .

[3] K. R. Dienes, C. F. Kolda, and J. March-Russell, Nucl. Phys. B492, 104 (1997), hep-ph/9610479.

[4] C. Cheung, J. T. Ruderman, L.-T. Wang, and I. Yavin (2009), 0902.3246.
[5] D. E. Morrissey, D. Poland, and K. M. Zurek (2009), 0904.2567.

[6] A. Katz and R. Sundrum (2009), 0902.3271.

[7] O. Adriani et al. (PAMELA) (2008), 0810.4995.

[8] J. Chang et al., Nature 456, 362 (2008).

[9] A. A. Abdo et al. (The Fermi LAT) (2009), 0905.0025.

[10] F. Aharonian et al. (H.E.S.S.), Phys. Rev. Lett. 101, 
261104 (2008), 0811.3894

[11] H. E. S. S. C. F. Aharonian (2009), 0905.0105.

[12] N. Arkani-Hamed, D. P. Finkbeiner, T. R. Slatyer, and N. Weiner, Phys. Rev. D79, 015014 (2009), 0810.0713.

[13] M. Pospelov and A. Ritz, Phys. Lett. B671, 391 (2009), 0810.1502 .

[14] J. Hisano, S. Matsumoto, and M. M. Nojiri, Phys. Rev. Lett. 92, 031303 (2004), hep-ph/0307216.

[15] J. March-Russell, S. M. West, D. Cumberbatch, and D. Hooper, JHEP 07, 058 (2008), 0801.3440.

[16] M. Cirelli, M. Kadastik, M. Raidal, and A. Strumia, Nucl. Phys. B813, 1 (2009), 0809.2409.

[17] I. Cholis, G. Dobler, D. P. Finkbeiner, L. Goodenough, and N. Weiner (2008), 0811.3641.

[18] I. Cholis, D. P. Finkbeiner, L. Goodenough, and N. Weiner (2008), 0810.5344.

[19] Y. Cui, D. E. Morrissey, D. Poland, and L. Randall, JHEP 05, 076 (2009), 0901.0557.

[20] D. Tucker-Smith and N. Weiner, Phys. Rev. D64, 043502 (2001), hep-ph/0101138.

[21] R. Bernabei et al., Int. J. Mod. Phys. D13, 2127 (2004), astro-ph/0501412.

[22] R. Bernabei et al. (DAMA), Eur. Phys. J. C56, 333 (2008), 0804.2741.

[23] S. Chang, G. D. Kribs, D. Tucker-Smith, and N. Weiner (2008), 0807.2250.

[24] D. Alves, S. R. Behbahani, P. Schuster, and J. G. Wacker (2009), 0903.3945.

[25] R. Essig, N. Sehgal, and L. E. Strigari (2009), 0902.4750.

[26] A. Arvanitaki et al. (2008), 0812.2075.

[27] B. Majorovits et al. (2004), astro-ph/0411396.

[28] J. Angle et al. (XENON), Phys. Rev. Lett. 100, 021303 (2008), 0706.0039.

[29] R. Hasty (XENON), AIP Conf. Proc. 842, 992 (2006).

[30] L. W. Kastens, S. B. Cahn, A. Manzur, and D. N. McKinsey (2009), 0905.1766.

[31] D. P. Finkbeiner, T. Lin, and N. Weiner (2009), 0906.0002

[32] R. Essig, P. Schuster, and N. Toro (2009), 0903.3941.

[33] B. Batell, M. Pospelov, and A. Ritz (2009), 0903.0363.

[34] M. Reece and L.-T. Wang (2009), 0904.1743.

[35] F. Bossi (2009), 0904.3815.

[36] P.-f. Yin, J. Liu, and S.-h. Zhu (2009), 0904.4644.

[37] M. Baumgart, C. Cheung, J. T. Ruderman, L.-T. Wang, and I. Yavin, JHEP 04, 014 (2009), 0901.0283.

[38] K. J. Kim and Y.-S. Tsai, Phys. Rev. D8, 3109 (1973).

[39] Y.-S. Tsai, Rev. Mod. Phys. 46, 815 (1974).

[40] Y.-S. Tsai, Phys. Rev. D34, 1326 (1986).

[41] C. Amsler et al. (Particle Data Group), Phys. Lett. B667, 1 (2008).

[42] B. Aubert et al. (BABAR) (2009), 0902.2176.

[43] M. Pospelov (2008), 0811.1030.

[44] J. D. Bjorken et al., Phys. Rev. D38, 3375 (1988).

[45] E. M. Riordan et al., Phys. Rev. Lett. 59, 755 (1987).

[46] A. Bross et al., Phys. Rev. Lett. 67, 2942 (1991).

[47] M. S. Turner, Phys. Rev. Lett. 60, 1797 (1988).

[48] N. Iwamoto, Phys. Rev. Lett. 53, 1198 (1984).

[49] A. A. Prinz et al., Phys. Rev. Lett. 81, 1175 (1998), hepex/9804008.

[50] F. Bergsma et al. (CHARM), Phys. Lett. B157, 458 (1985).

[51] B. A. Mecking et al. (CLAS), Nucl. Instrum. Meth. A503, 513 (2003).

[52] A. Afanasev et al. (2008), 0810.4189.
[53] A. Afanasev et al., Phys. Rev. Lett. 101, 120401 (2008), 0806.2631.

[54] D. Hooper and T. M. P. Tait (2009), 0906.0362.

[55] J. Mardon, Y. Nomura, and J. Thaler (2009), 0905.3749.

[56] Y. Nomura and J. Thaler (2008), 0810.5397.

[57] M. J. Strassler and K. M. Zurek, Phys. Lett. B651, 374 (2007), hep-ph/0604261.

[58] We thank Takashi Maruyama for providing calculations of downstream particle densities using FLUKA. 